\title{
Constitutive loss of DNMT3A causes morbid obesity through misregulation of adipogenesis
}

Ayala Tovy ${ }^{1,2}$, Jaime M. Reyes ${ }^{1,2,3}$, Linda Zhang ${ }^{1,2,4}$, Yung-Hsin Huang ${ }^{1}$, Carina Rosas ${ }^{1,2}$, Alexes C. Daquinag ${ }^{5}$, Anna G. Guzman ${ }^{1,2}$, Raghav Ramabadran ${ }^{1,2}$, Chun-Wei Chen ${ }^{1,2,3}$, Tianpeng Gu ${ }^{1,2}$, Sinjini Gupta ${ }^{1,2}$, Laura Ortinau, ${ }^{3,6}$, Dongsu Park ${ }^{3,6}$, Aaron R. Cox ${ }^{2,7}$, Rachel E Rau ${ }^{1,8}$, Sean M. Hartig $^{2,7}$, Mikhail G Kolonin ${ }^{4}$ and Margaret A. Goodell ${ }^{1,2,3,5^{*}}$

1. Stem Cells and Regenerative Medicine Center, Baylor College of Medicine, Houston, TX, USA

2. Department of Molecular and Cellular Biology, Baylor College of Medicine, Houston, TX, USA

3. Department of Molecular and Human Genetics, Baylor College of Medicine, Houston, TX, USA

4. Graduate Program in Translational Biology and Molecular Medicine, Baylor College of Medicine, Houston, TX, USA

5. Institute of Molecular Medicine, McGovern Medical School at the University of Texas Health Science Center, Houston, TX, 77030, USA

6. Center for Metabolic and Degenerative Disease, Institute of Molecular Medicine, McGovern Medical School, University of Texas Health Science Center at Houston, Houston, TX, USA

7. Division of Endocrinology, Diabetes, and Metabolism, Department of Medicine, Baylor College of Medicine, Houston, TX, USA

8. Department of Pediatrics, Baylor College of Medicine and Texas Children's Hospital, Houston, TX, USA.

* To whom correspondence should be addressed

goodell@bcm.edu

713-798-1265 


\section{Abstract}

DNA Methyltransferase 3A (DNMT3A) is an important facilitator of differentiation of both embryonic and hematopoietic stem cells. Heterozygous germline mutations in DNMT3A lead to Tatton-Brown-Rahman Syndrome (TBRS), characterized by obesity and excessive height. While DNMT3A is known to impact feeding behavior via the hypothalamus, here we investigated a role in adipocyte progenitors utilizing heterozygous knockout mice that recapitulate cardinal TBRS phenotypes. These mice become morbidly obese due to adipocyte enlargement and tissue expansion. Adipose tissue in these mice exhibited defects in preadipocyte maturation and precocious activation of inflammatory gene networks, including interleukin-6 signaling. Adipocyte progenitor cell lines lacking DNMT3A exhibited aberrant differentiation. Furthermore, mice in which Dnmt3a was specifically ablated in adipocyte progenitors showed enlarged fat depots and increased progenitor numbers, partly recapitulating the TBRS obesity phenotypes. Loss of DNMT3A led to constitutive DNA hypomethylation, such that the DNA methylation landscape of young adipocyte progenitors resemble that of older wild-type mice. Together, our results demonstrate that DNMT3A coordinates both the central and local control of energy storage required to maintain normal weight and prevent inflammatory obesity. 


\section{Introduction}

De novo DNA methyltransferase 3A (DNMT3A) plays a central role in establishing DNA methylation patterns during mammalian early embryogenesis and development (Okano et al., 1999). Deletion of Dnmt3a in mice results in postnatal lethality, confirming its necessity during development (Li et al., 1992). In humans, somatic mutations in DNMT3A are enriched in the hematopoietic system during aging and malignancy (Ley et al., 2010; Tovy et al., 2020). Loss of DNMT3A in hematopoietic stem and progenitor cells alters their epigenetic landscape, increasing the stem cell pool through self-renewal at the expense of differentiation (Challen et al., 2011). Whether DNMT3A regulates differentiation in other stem cell populations is largely unknown.

Germline heterozygous mutations in DNMT3A lead to Tatton-Brown-Rahman syndrome (TBRS; OMIM: 615879) (Tatton-Brown et al., 2014), a dominant disorder characterized by excessive height ( $80 \%$ of individuals), intellectual disability ( $80 \%$ of individuals), and obesity ( $70 \%$ of individuals) (Tatton-Brown et al., 2014; Tatton-Brown et al., 2018). We and others have recently shown that $D n m t 3 a$ haploinsufficent mice can be used to study TBRS (Christian et al., 2020; Tovy et al., 2021). These mice recapitulate key TBRS phenotypes, including enlarged body size and obesity (Christian et al., 2020). However, the mechanisms through which DNMT3A regulates weight gain in TBRS are incompletely understood.

Obesity is a complex energy balance disorder reflecting deficient communication between the hypothalamus and adipose tissue, and only a few genes have so far been identified in which mutations exert causal roles in humans (Choquet and Meyre, 2010). Of these genes, the majority play a role in the central nervous system, which is pivotal in regulating behaviors controlling nutrient update and energy expenditure. Yet, although it is well known that mechanisms that impact adipocyte progenitor expansion and differentiation will also lead to obesity, the data on a potential role for DNA methylation in regulating adipose tissue are conflicting (Ma and Kang, 2019). While broad inhibition of DNA methyltransferases with compounds such as 5-aza-2'-deoxycytidine was reported to promote lipid accumulation in preadipocyte cell lines (Yang et al., 2016), DNMT inhibition in preadipocyte and mesenchymal precursor cell lines reduced adipogenic differentiation capacity (Chen et al., 2016b). Similarly, the role of $D N M T 3 A$ in body weight regulation is unclear. While one study showed homozygous ablation of Dnmt3a specifically in hypothalamic Sim1-positive neurons led to hyperphagia and obesity (Kohno et al., 2014), knockout of Dnmt3a in mature adipocytes protected mice from developing insulin resistance while on a high-fat-diet (You et al., 2017). 
Here, we utilize a mouse model of TBRS to establish that while dysregulation of Dnmt3a indeed regulates food intake, Dnmt3a also plays a cell-intrinsic role in adipocyte progenitors to permit normal maturation. We find that, similar to its role in the hematopoietic system, DNMT3A regulates adipose stem and progenitor cell function. Our results establish that DNMT3A directly regulates obesity through the adipose tissue, in addition to its role in the hypothalamus, and demonstrate how constitutive loss of DNMT3A creates a domino effect in which excess feeding together with faulty adipocytes lead to obesity.

\section{Results}

\section{Heterozygous Dnmt3a loss leads to adipose tissue expansion and weight gain}

TBRS patients harbor heterozygous frameshift or missense mutations in DNMT3A, predicted to be partial or complete loss-of-function, distributed throughout the gene's main functional domains (Tatton-Brown et al., 2018; Tovy et al., 2021). In order to study the role of DNMT3A in weight gain and adipose tissue biology, we utilized mice heterozygous for a Dnmt3a null allele ("3A-HET") along with their wild-type (WT) counterparts. In humans, TBRS phenotypes become particularly evident around adolescence when patients exhibit marked height and weight gain (Tatton-Brown, 2018). Similarly, WT and 3A-HET mice were indistinguishable until around 3 months of age. However, by 6 months, both male and female 3A-HET mice were heavier than controls (Fig 1A and Supplemental Fig. 1A). At 12 months of age, the cohort of HET averaged 3 standard deviations (SD) above the mean weight of WT mice, becoming obese (Fig. 1B-C). The increased length of 3A-HET mice, while statistically significant, was mild, and therefore could not account for the increased weight (Supplemental Fig. 1B). Body composition analysis was performed to determine whether fat versus lean mass contributed to the weight difference. We detected a steady increase in fat percentage and a concomitant reduction in lean mass in 3A-HET mice (Fig. 1 C-D, Supplementary Fig. 1G).

Adipose tissue can expand through either increased number of adipocyte cells due to proliferation and differentiation of adipocyte precursors (hyperplasia) or increased adipocyte cell size (hypertrophy). White adipose tissue (WAT) functions primarily to store energy, while brown adipose tissue (BAT) performs thermogenic functions. From WT and 3A-HET mice at one year of age, we dissected fat depots (gonadal, inguinal white adipose tissue and brown adipose tissue) and performed histological analysis to determine size of BAT and WAT adipocytes. On average, 3A-HET mice had heavier fat depots (Supplementary Fig. 1H) and larger adipocytes in 
both WAT and BAT depots compared to WT littermates (Fig. 1E-F), suggesting the increase in total fat is largely due to hypertrophy. These results suggest that reduced DNMT3A leads to pathological adipose expansion and spontaneous obesity.

Fatty liver is strongly associated with obesity, we therefore performed histological analysis of WT and 3A-HET mice livers at one year of age. We observe increase in fat accumulation in 3A-HET livers, as measured by fat-soluble dye (Oil-Red-O staining). Therefore, these results demonstrate that germline loss of DNMT3A leads to weight gain through expansion of adipose tissue, as well as accumulation of lipids in non-adipose tissues such as the liver (Fig. G-H).

Together these data from heterozygous Dnmt3a KO mice mirror the findings in TBRS patients of increased size and marked obesity that develops after adolescence. Thus, we further examined the mechanisms accounting for obesity associated with reduced DNMT3A.

\section{Dnmt3a-deficient mice are hyperphagic and develop glucose and insulin resistance}

We next tested whether 3A-HET mice showed increased food intake or decreased energy expenditure, or both, using a comprehensive lab animal monitoring system (CLAMS). We profiled WT and HET males and females at 6 months of age (Supplementary Fig. 2a). We measured increased food intake in HET mice mostly during the dark phase (Fig. 2A). HET mice also showed a mild change in activity during light phase and decrease in energy balance calculated by calorie consumption compared to calorie expenditure (Fig. 2B and Supplemental Fig. 2C). Other metabolic parameters were not altered (Supplemental Fig. 2B-F). As leptin secreted from adipocytes normally signals satiety to the hypothalamus to restrict food intake and inhibit weight gain (Badman and Flier, 2007; Rosen and Spiegelman), we measured leptin levels in 3A-HET compared to WT mice. We observed a persistent increase in leptin levels (Supplemental Fig. 2G) in mice lacking DNMT3A compared to their WT littermates. These data demonstrate that heterozygous germline loss of Dnmt3a impacts feeding behavior similarly to homozygous loss of Dnmt3a specifically in the hypothalamus (Kohno et al., 2014).

Prolonged weight gain can result in insulin resistance, which manifests as hyperinsulinemia combined with hyperglycemia and dyslipidemia. Thus, we tested whether 3AHET mice displayed insulin resistance before (2 months) and after (6 months) weight gain. At 2 months, WT and HET mice did not display major differences in fasting glucose and insulin levels or when challenged with a glucose tolerance test (Fig 2. C-D and Supplementary Fig. 2H). However, at 6 months, when 3A-HET mice are statistically heavier than WT, they had higher fasting levels of glucose and insulin (Fig. 2D). Further, at 10 months HET mice develop 
profound glucose and insulin resistance (Fig. 2E). These results establish that loss of a Dnmt3a in this mouse model of TBRS leads to obesity and contributes to insulin resistance at older ages.

\section{DNMT3A promotes adipocyte differentiation and its loss increases progenitor pool}

Given the marked obesity of Dnmt3a heterozygous mice, we considered the possibility that factors other than hypothalamus-regulated behaviors contribute additively to the increased adiposity. Because loss of DNMT3A can inhibit somatic stem cell differentiation (Challen et al., 2011), we scrutinized adipocyte maturation in 3A-HET mice.

Adipose tissue depots are composed of multiple cell populations including preadipocyte stem and progenitor cells, preadipocytes, and fully mature adipocytes, which change during development (Merrick et al., 2019). To examine how loss of Dnmt3a affected these cell populations, we performed single-cell RNA sequencing (scRNAseq) on cells isolated from inguinal white adipose tissue (iWAT) of WT and 3A-HET mice at 8-weeks and 1-year old. We selected these time points to capture adipose tissue cell states that span an expansion period (8 weeks) and obesity in 3A-HET mice (1 year), reflecting changes in adipose tissue and weight gain over time. We focused on WAT since its associated with increased risk for insulin resistance as observed in $3 \mathrm{~A}-\mathrm{HET}$ mice.

We first defined profiles in WT adipose tissue focusing on preadipocyte stem and progenitors. We separated stromal vascular cells (SVCs) from adipose tissue and then used flow cytometry to sort adipocyte stem and progenitor cells (Fig. 3A and Supplementary Fig. 3A) and subjected those cells to scRNAseq. We performed standardized normalization and principal component analysis (PCA) on all conditions and from both genotypes (Supplementary Fig. 3BC). To define component cell types, we next performed unsupervised clustering of gene expression of all cells and obtained six clusters (Fig. 3B). We defined cluster marker genes as those differentially expressed in each cluster compared to all other clusters (Supplementary Table 1, Fig. 3C-D) and used those marker genes to define cluster identity using MSigDB pathway enrichment. Cluster 1 expressed key genes associated with committed preadipocytes, adipocyte differentiation, and inflammatory activation such as fatty acid binding protein (Fabp4), lipoprotein lipase ( $L p /)$, and intercellular adhesion molecule-1 (Icam1) (Balamurugan and Sterneck, 2013). Cluster 2 displayed markers of committed differentiating preadipocytes (Fabp4, Lpl and Plin2), as well as enrichment for the tissue factor F3, a marker of adipogenesisregulatory cells associated with adipocyte differentiation (Schwalie et al., 2018). 
We observed that both clusters 3 and 4 expressed a mix of stem and progenitor markers (Dpp4, Cd34) and adipocyte markers (Fabp4, LpI), indicating they contained cells in transition. Importantly, dipeptidyl peptidase (DPP4) is a marker of multipotent progenitors within iWAT with some attributes of mesenchymal stem cells (Merrick et al., 2019). Accordingly, pathway enrichment analysis of clusters 3 and 4 identified hallmarks of "epithelial to mesenchymal transition" and "adipogenesis" (Fig. 3C-D, Supplementary Fig. 3A). However, cluster 3 uniquely had increased expression of pro-inflammatory markers. Clusters 5 and 6 harbored gene profiles of multipotent stem and progenitor cells (Dpp4, Cd34, Ly6a (encoding Sca-1), and Pi16) and did not express differentiated adipocyte markers such as Fabp4; most of these markers displayed higher expression in cluster 5 than cluster 6 (Fig. 3B-C, Supplementary Fig. 3A). With these data and the gradient in Dpp4 and Fabp4 expression across clusters (Fig. 3E), we classified three major groups of cells: committed preadipocytes (COM; clusters 1-2, with high Fabp4), primed preadipocytes (PRI; (clusters 3-4, with both stem-like and differentiation markers), and stem and progenitor clusters (ST; clusters 5-6).

We next examined how the cellular composition of WT and 3A-HET adipose tissue changed over time. We calculated the proportion of WT and 3A-HET cells in each of these cellular states at 8-weeks and after 1 year. We found that at young ages, the proportions of stem and primed preadipocytes progenitors were higher in 3A-HET mice (1.35- and 1.5-fold, respectively) compared to WT, while the proportion of cells in the committed clusters was lower than in WT (1.4- fold) (Fig. 3F, Supplementary Fig. 3E). These differences in the stem and progenitor-primed clusters indicate that young $3 \mathrm{~A}-\mathrm{HET}$ mice harbor a larger pool of stem and progenitor cells at the expense of committed adipocytes.

After one year, when $3 \mathrm{~A}-\mathrm{HET}$ mice are obese, cells in committed clusters decreased proportionally in WT but increased in 3A-HET iWAT (Fig. 3F). In addition, we detected a decrease in the ST stem cluster in 1-year old 3A-HET compared to WT mice, suggesting that adipose expansion in HET mice ultimately results in a decrease in the stem and progenitor clusters, possibly due to exhaustion or increased proliferation.

To validate these observations in cellular proportions, we examined changes with age in immunophenotypically defined progenitors. Consistent with our scRNAseq data, we found that, though preadipocyte cells were more abundant in 3A-HET mice at 8-weeks relative to WT, by one year their numbers decreased relative to WT (Fig. 3G). These results imply that intermediate stem and primed adipocytes are the primary source for adipose tissue expansion in WT mice, while in 3A-HET mice, increased energy intake and hyperphagia continuously require more preadipocyte progenitors, ultimately resulting in depletion of stem cells. 


\section{Loss of DNMT3A leads to inflammatory obesity}

To better understand which cell types differed in 3A-HET mice and how they changed over time, we used pseudotime analysis of the scRNA-seq data to infer relationships among the cell type clusters. This analysis revealed two major trajectories (A and $B$ ), with cluster 5 representing the most naïve position (Fig. 4a-b). Trajectory A included stem-like cluster 6, and primed (cluster 4) and committed preadipocytes (cluster 2, Fabp $4^{\text {high }}$ ). Trajectory B passed through clusters that displayed hallmarks of the inflammatory response including upregulated interleukin 6 (I/6) and Ccl2 (C-C motif chemokine ligand 2) (Fig. 3B, 6C, Supplementary Fig. 4A). Trajectory B also included the pro-inflammatory primed (cluster 3) ending in committed preadipocytes (cluster 1).

Next, we calculated the proportion of WT and $3 A-H E T$ cells residing along each of the trajectories. Young WT cells were weighted toward trajectory A, while young 3A-HET cells were much more heavily represented along the pro-inflammatory $B$ trajectory, with more cells retaining stem-like and primed preadipocyte phenotypes (Fig. 4D). Importantly, at 8 weeks of age, WT and 3A-HET mice did not display overt differences in adiposity, indicating these cellstate differences precede the phenotypic alterations.

Examining the trajectories after 1 year, we noted in WT mice more cells in the proinflammatory path, consistent with the known inflammatory effects of aging in adipose tissue (Tchkonia et al., 2010). Strikingly, the 3A-HET preadipocytes showed a particularly strong bias toward pro-inflammatory trajectory B (Fig. 4D). Altogether, these analyses suggest that cluster 5 serves a source for both pathways, and 3A-HET adipose tissue, even at a young age, displays a bias towards an inflammatory state, which becomes exacerbated in aging obese preadipocytes.

Because the inflammatory state of adipose tissue correlates strongly with insulin resistance and other co-morbidities of obesity, we examined the inflammatory markers and trajectories more closely. Differential gene expression analysis supported our observation that 3A-HET cells were primed to express higher pro-inflammatory marker genes in the transient and committed preadipocytes groups (Fig. 4E, Supplementary Fig. 4B-C). Moreover, 3A-HET preadipocytes displayed activation of inflammatory signaling in the IL6 and interferon gamma signaling pathways even in the stem-like cells (Supplementary Fig. 6B). To verify that IL6 was upregulated, we performed intracellular flow cytometry analysis of preadipocytes from WT and 3A-HET mice (6 months of age), confirming markedly elevated IL6 (Fig. 4F).

Along with the $3 \mathrm{~A}-\mathrm{HET}$ inflammatory phenotype, genes representing full differentiation of adipocytes (Apoe, Lpl, Fabp4, and lgfbp3) were expressed at lower levels in 3A-HET cells in the 
primed and committed cell clusters relative to that of WT tissue at both time points (Supplementary Fig. 4d). In addition, unlike WT cells which displayed an epithelial to mesenchymal transition (EMT) signature only in the stem cell clusters, 3A-HET tissue displayed enrichment of stem-like EMT even in the primed and committed clusters, suggesting a failure to suppress the stem cell program in $3 \mathrm{~A}-\mathrm{HET}$ preadipocytes (Fig. 4E). Together, these results support a role for DNMT3A in adipocyte precursor cell differentiation and maturation. Our data also indicate that even heterozygous loss of Dnmt3a in preadipocytes promotes proinflammatory obesity.

\section{DNMT3A promotes differentiation of preadipocyte progenitor cell lines}

Our single cell analysis showed that differentiating 3A-HET preadipocytes upregulate inflammatory pathways, while improperly activating adipogenesis. However, concomitant absence of DNMT3A in the hypothalamus and the adipose tissue in germline Dnmt3a KO mice confounds interpretation of a specific role for DNMT3A in adipocyte progenitors. Therefore, here we sought to identify if loss of DNMT3A resulted in similar transcriptional and phenotypic consensuses in WAT and BAT preadipocyte cell lines. Therefore, we generated Dnmt3aknockout (KO) clonal cell lines in established white (3T3-L1) and brown (BAC-C4) preadipocyte models (Fig. 5A and Supplementary Fig. 5A).

First, we compared growth rates of 3T3-L1-control and 3T3-L1-Dnmt3a-KO (3T3-Con and 3T3-KO, hereafter) and of BAC-C4 control and BAC-C4-Dnmt3a-KO (BAC-Con and BAC$\mathrm{KO}$ ). In both lineages, Dnmt3a-KO clones exhibited higher proliferation rates than controls (Fig. $5 \mathrm{~B}$ and Supplementary Fig. 5B), mirroring the expansion of $3 \mathrm{~A}-\mathrm{HET}$ progenitor cells in our scRNAseq analysis. In response to hormonal stimulation, 3T3-L1 and BAC-C4 terminally differentiate into adipocytes that accumulate triglycerides in the form of lipid droplets. To analyze the role of DNMT3A in adipocyte differentiation, we quantified lipid droplet size and number in our control and knockout lines. We found that all four lines differentiated and formed mature adipocytes that stored lipids, but both 3T3-KO and BAC-KO stored fewer lipids (Fig. 5C and Supplementary Fig. 5C). In addition, both $\mathrm{KO}$ cell lines accumulated fewer lipid droplets when challenged with exogenous fluorescent fatty acids, indicating that loss of DNMT3A impairs lipogenesis (Fig. 5D and Supplementary Fig. 5D).

In addition to lipogenesis, adipocytes breakdown lipids through lipolysis (Ertunc and Hotamisligil, 2016). To test the role of DNMT3A in lipolysis, we treated WT and KO cell lines with isoproterenol, a non-selective $\beta$-adrenergic receptor agonist. We observed slowed release of fatty acids in both $\mathrm{KO}$ lineages with no effect on cell numbers (Fig. 5E, Supplementary Fig. 
$5 \mathrm{E})$. Moreover, phosphorylation of the key lipase regulator hormone sensitive lipase (HSL) was significantly reduced in both $\mathrm{KO}$ lineages (Fig. 5F, Supplementary Fig. 5F), consistent with reduced lipolysis. Combined, these results indicate loss of DNMT3A in preadipocytes allows at least partial differentiation but impairs both lipogenesis and lipolysis responses in WAT and BAT.

To broadly examine the DNMT3A-regulated differentiation program in BAT, we performed RNAseq on BAC-Con and BAC-KO cells and identified differentially expressed genes in non-treated (NT), differentiated, and isoproterenol treated cells (ISO). We identified $\sim 1000$ genes that were significantly differentially expressed between BAC-Con and BAC-KO cells following differentiation (Supplementary Fig. $5 \mathrm{G}$ and Supplementary Table 2). When we classified genes upregulated in KO compared to control following differentiation or ISO treatment, we noticed that 30\% (244 genes, Supplementary Fig. 5G left) were already differentially expressed in NT cells (Fig. 3G top). In contrast, only a small number (41 genes, Supplementary Fig. 5G right) of genes that were upregulated in control cells following differentiation were already altered at baseline in $\mathrm{KO}$ cells.

When we plotted the average expression of genes differentially expressed in control or $\mathrm{KO}$ cells following 5 days differentiation, these genes were broadly altered in the same direction in the untreated KO cells (Fig. 5G-H). The large number of genes not downregulated in KO cells during differentiation indicates that DNMT3A is needed for gene repression during adipocyte differentiation. Pathway enrichment analysis showed that while genes upregulated in control cells during differentiation were enriched for adipogenesis, $\mathrm{KO}$ cells displayed enrichment for EMT and inflammation (Fig. 5G-H).

To determine if loss of DNMT3A similarly effected gene expression in WAT, we examined data from differentiating 3T3-L1 cells following Dnmt3a knock-down (You et al., 2017). Genes upregulated in BAC-KO significantly overlapped with those in 3T3-L1 siRNADnmt3a (163 genes, Supplementary Fig. 5H top). Pathway enrichment of overlapping genes differentially expressed due to Dnmt3a loss in BAC also indicated congruence with in the 3T3L1 cell line, including upregulation of EMT and inflammation (Supplementary Fig. $5 \mathrm{H}$ bottom; Supplementary Table 3). The EMT gene signature is consistent with a mesenchymal stem cell state, indicating that loss of DNMT3A causes both BAT and WAT to retain a preadipocyte-like gene expression pattern despite at least partial induction of differentiation. These data establish a cell-autonomous role for DNMT3A in maturation of both white and brown adipocytes.

\section{Loss of DNMT3A perturbs similar transcriptional pathways in vivo and in vitro}


To gain further support for an in vivo role for DNMT3A in adipocyte maturation, we sought to compare the changes observed in WAT and BAT cell lines lacking Dnmt3a with changes observed in our single-cell data from adipocyte progenitors. We performed GSEA analysis and overlapped the normalized enrichment score (NES) from both datasets (Supplemental Table 4-5). We compared the undifferentiated BAC progenitors to the in vivo population of cells we identified as "primed", and the differentiated BAC progenitors to the "committed" populations as identified for WT and HET in the scRNAseq (Fig. 3E). Our results show that key gene hallmarks differentially expressed in the preadipocyte cell lines (BAC and 3T3) include gene sets identified in our single cell data, such as inflammatory, IL6 and epithelial mesenchymal transition (Fig. 6A-B). Expression of these gene pathways is consistent with incomplete differentiation and upregulation of inflammation following loss of DNMT3A both in vitro and in vivo.

Our cell line data indicate that DNMT3A is required for induction of a functional lipolysis program. Regulation of lipolysis is important to maintain energy levels during fasting. To determine whether impaired lipolysis impacted free fatty acid (FFA) release in serum, we fasted 10 month-old WT and 3A-HET mice for 12 hours. We detected less FFA and glycerol in the plasma in 3A-HET mice compared to WT ( 2 fold; Fig. 6 C-D). This also correlated with decreased pHSL (Supplemental Fig. 6A). To address the role of DNMT3A directly in lipolysis, we also stimulated lipolysis ex vivo with ISO treatment of adipose fat pads extracted from 6month-old WT and HET mice and measured release of FFA and glycerol. 3A-HET tissues displayed lower secretion of FFA from both BAT and WAT (gonadal, gWAT) compared to WT (Fig. 6E, Supplemental Fig. 6E). In addition, 3A-HET WAT and BAT tissues released less glycerol compared to WT (Fig. 6F, Supplemental Fig. 6C). Consistent with our in vitro data, western blot analysis of 3A-HET fat depots showed diminished activation of p-HSL compared to WT in response to ISO or fasting (Supplementary Fig. 6D-E). Together, these findings demonstrate that, similar to homozygous loss of Dnmt3a in cell lines, heterozygous loss of Dnmt3a in vivo reduces lipolytic capacity and subsequent FFA release.

\section{Ablation of Dnmt3a in subcutaneous preadipocytes leads to adipose tissue expansion}

Our results thus far suggest a direct role for DNMT3A in regulation of adipose tissue formation. To further test for a cell autonomous role for DNMT3A in adipocyte development, we conditionally ablated Dnmt3a using transgenic mice expressing Cre under the control of the Prx enhancer (Logan et al., 2002). Prx is expressed in the uncommitted mesenchymal progenitors 
of subcutaneous inguinal fat, which were the focus of the analyses described above (Eguchi et al., 2011; Logan et al., 2002).

We monitored mice that were either control (Dnmt3a $a^{\text {floxflox }}$ ) or homozygous Dnmt3a knockout (PRX-D3A) in inguinal fat for 8 months. We observed a steady yet mild increase in weight ( 14\%), which became statistically significant at 8 months in PRX-D3A mice (Fig. 7A and Supplementary Fig. 7A-B). Body composition analysis confirmed that PRX-D3A mice displayed elevated fat mass compared to controls (Fig. 7B). We also detected an increase in plasma leptin in PRX-D3A mice consistent with expansion of adipose tissue in these mice (Fig. 7C). These results support our hypothesis that loss of DNMT3A specifically from adipocyte progenitors contributes to expansion of adipose tissue mass.

Prx-Cre is expressed in inguinal fat and not visceral fat (Krueger et al., 2014). Thus, if DNMT3A acts autonomously in adipocyte progenitors, we expected to observe expansion of the adipocyte progenitor pool in inguinal but not visceral fat depots. We performed flow cytometry analysis of SVCs extracted from fat pads of subcutaneous inguinal and visceral fat and indeed observed a significant increase in the percentage of Sca1+ preadipocytes progenitors only from the subcutaneous inguinal fat, supporting cell autonomous expansion of adipose progenitors with loss of DNMT3A (Fig. 7D). We also measured the efficacy of lipolysis in subcutaneous inguinal fat from PRX-D3A mice. Similarly to our results from D3A-HET mice, we observed a decrease in the release of FAA from the PRX-D3A fat pads (Fig. 7E). Taken together, these results confirm a role for DNMT3A in regulation of obesity via early stages of adipogenesis.

\section{DNMT3A-mediated DNA methylation is required for adipose tissue development}

To examine how loss of DNMT3A contributes to functional changes in preadipocytes, we examined DNA methylation alterations using whole genome bisulphite sequencing (WGBS) in SVCs at the same time points used for scRNAseq (8 weeks and 1 year). In 3A-HET cells, we observed a mild $(2-4 \%)$ yet significant $\left(p<10^{-15}\right)$ decrease in global methylation compared to WT cells in both time points (Supplementary Fig. 8A).

As obesity became extreme with age, we sought to compare age-associated changes in both WT and 3A-HET cells. We first determined DNA methylation changes in WT between the two time points, identifying $\sim 1100$ regions that were hypomethylated (hypo-DMRA, see methods), and $\sim 2800$ regions hypermethylated with age (hyper-DMRA, see methods) (Supplementary Fig. 8B, Supplementary Tables 6-7). We then examined the mean methylation 
distribution of WT hypo-DMRA in 3A-HET cells (Fig. 8A). Remarkably, these regions tended to be hypomethylated in $3 \mathrm{~A}-\mathrm{HET}$ cells regardless of age (Fig. 8B). Comparing the mean methylation for these regions between young WT and HET cells showed that 900 of them $(70 \%)$ displayed lower methylation values ( $\geq 19 \%$, blue peak in Fig. $8 \mathrm{C})$ in HET cells than WT and most remained hypomethylated at 1 year (Fig. 8D and Supplementary Fig. 8C). Therefore, 3A-HET cells lacking one allele of Dnmt3a began with lower DNA methylation and failed to experience the dynamic methylation changes typically seen in WT cells with age.

We next examined the role of DNA methylation gains over time, focusing on the WT hyper-DMRA (Fig. 8e). When we plotted their mean methylation in 3A-HET cells, young cells displayed significantly lower methylation levels than WT, and methylation was generally even lower at 1 year ( 1500, 54\%). These data suggest that DNMT3A has a major role in methylation of ageing DMRA regions over time (Fig. 8F). In fact, when we plotted the methylation of the hyper-DMRA in young and old 3A-HET cells, most of them ( 1200) remained hypomethylated across time (Fig. 8G, purple and Supplementary Fig. 8C). Taken together, these results suggest that DNMT3A is essential to modulate the DNA methylation landscape during adipocyte development and aging, and that even heterozygous loss of Dnmt3a blunts these dynamics.

\section{Loss of DNMT3A induces a pro-inflammatory trajectory in young adipocytes}

To identify genes influenced by loss or gain of DNA methylation in WT and 3A-HET cells, we performed gene ontology analysis using Hypergeometric Optimization of Motif EnRichment (HOMER), analyzing hypo-DMRA and hyper-DMRA regions (Fig. 8). Our analysis revealed that hypo-DMRA were largely associated with inflammatory and metabolic pathways, while hyper-DMRA were enriched for pathways associated with stem cells (Supplementary Fig. 9A-B).

In the absence of DNMT3A, lower methylation reflects a failure to establish DNA methylation. Our cell lines and scRNAseq work revealed that 3A-HET cells fail to properly upregulate the adipogenesis gene program while aberrantly increasing a pro-inflammatory cell trajectory. Because WT hypo-DMRA were associated with pro-inflammatory pathways, we hypothesized that lower DNA methylation in those regions in young HET cells would correlate with higher activation of pro-inflammatory trajectory $\mathrm{B}$. We therefore compared gene expression of stem, primed, and committed cell clusters in trajectories A or B separated by genotype to determine in which cell clusters hypo-DMRA led to upregulation of gene expression (average of log2 fold-change) (Fig. 9A). We detected significant correlation for the 3A-HET primed pre- 
adipocytes, and weaker correlation for committed pre-adipocytes (Fig. 9B. These results indicate that pro-inflammatory-associated regions that lose methylation during WT aging and are even less methylated in HET cells show increased gene expression in 3A-HET primed and committed cell clusters. Thus, reduced DNA methylation in those regions permits the activation of the pro-inflammatory genes. Examples of genes with this behavior include Irf1, Socs3 and Jak2 (Supplementary Fig. 9C). Activation of all these genes in adipocytes previously has been associated with metabolic disease and inflammation (Friesen et al., 2017; Liu et al., 2015).

We found WT-defined hyper-DMRAs to be regions highly enriched for pathways associated with stem cell and pluripotency, suggesting that the stem cell program is repressed as WT adipose tissue ages and stem cells differentiate (Fig. 5F). We hypothesized that hyperDMRAs that did not gain methylation over time in HET cells would be associated with upregulated genes. Indeed, when we calculated the enrichment score of the gene expression profile from our scRNAseq of WT and HET at one year of age, HET cells displayed high correlation with activation of WT hyper-DMRA, which include known stromal progenitor genes such as F3, protein phosphatase 1 (DUSP1) and PDGF receptor $\beta$ (PDGFRB) (Fig. 9C-D). Interestingly, F3 is known for its role in inhibitory adipogenesis regulators (Schwalie et al., 2018), suggesting inhibited differentiation in HET cells. Overall, these results suggest that the failure to gain DNA methylation with age in obese mice promotes enduring expression of stem and progenitor genes, consistent with faulty adipocytes.

\section{Discussion}

Given the constitutional loss of DNMT3A that functions across multiple tissues, our TBRS model offers a unique opportunity to study the association between DNA methylation, adipogenesis, and obesity. Here, we establish a role for DNMT3A in regulating adipogenesis in vivo and in vitro and demonstrate that DNMT3A deficiency predisposes mice to obesity, even on a normal diet. We verified a role for DNMT3A in regulation of feeding and demonstrated for the first time a direct independent role for DNMT3A in regulating adipose tissue formation and function.

We show that heterozygous Dnmt3a-null mice recapitulate the obesity phenotype observed in TBRS along with increased length. Our studies show aberrant feeding behavior in HET mice which is in line with previously published data (Kohno et al., 2014). In view of the recently established studies on the role of DNMT3A in emotional behavior and anxiety (LaPlant et al., 2010; Morris et al., 2016), future studies may address the association between DNMT3A loss, complex neuro-psychiatric disorders and feeding behaviors. 
Here we report the particularly striking impact of reduced of DNMT3A on adipose tissue expansion and weight gain. Mutant mice displayed expanded fat depots of all types. Adipocytes were larger with decreased lipolysis as supported by phenotypic and transcriptomic analyses. The trajectory of weight gain is distinct from that of leptin-null mice, which become obese by 4 weeks (Zhang et al., 1994). Instead, heterozygous Dnmt3a KO mice gain weight gradually, with marked tissue expansion evident mostly after 6 months. Nevertheless, after around 1 year, the HET mice are markedly obese, with body weight typically about a third higher than their WT counterparts.

We present evidence here that, in addition to its role in energy homeostasis through the central nervous system, DNMT3A directly regulates fat by enabling proper adipocyte progenitor development. The increased fat after ablation of Dnmt3a specifically in inguinal depots supports a cell autonomous function for DNMT3A in preadipocytes. Furthermore, ablation of Dnmt3a in white and brown preadipocyte progenitor cell lines led to incomplete differentiation, underscoring the direct role. While knockout of Dnmt3a using Adiponectin-Cre, which is specific for fully differentiated adipocytes, did not lead to weight gain (You et al., 2017), this is expected given our hypothesis that DNMT3A primarily acts in stem and progenitor cells and not on differentiated cells. This concept was elegantly validated in the hematopoietic system where knockdown of DNMT3A in human macrophages had minimal effect compared to its loss in stem cells (Lim et al., 2021). These results support the broader concept that a primary role for DNMT3A is in progenitors, enabling proper differentiation, after which it is dispensable.

Our data indicate that loss of DNMT3A expands adipocyte progenitors which exhibit aberrant differentiation: young 3A-HET mice displayed an increase in the preadipocyte pool and fewer committed cells compared to WT, and differentiated cells in vivo and in vitro were less functional by multiple measures. Paradoxically, this led to more adipose tissue and obesity. While counterintuitive, these results are in accord with observations from the hematopoietic system. Loss of DNMT3A leads to a marked increase in stem cell pool. Despite somewhat impaired differentiation, the greater number of stem cells leads to an overall increase in the number of differentiated cells in the peripheral blood (Challen et al., 2011; Jeong et al., 2018). This imbalance between self-renewal and differentiation is believed to underlie the propensity of DNMT3A mutations to contribute to clonal hematopoiesis and leukemia (Challen and Goodell, 2020). 
In addition to blunted differentiation, loss of DNMT3A led to a precocious inflammatory phenotype in the adipose tissue. We used scRNAseq and DNA methylation analysis to define features of white adipose tissue over time in WT and HET mice. Similar to previously published scRNAseq studies (Merrick et al., 2019), we identified stem-like DPP4 ${ }^{\text {high }}$ cells as a renewable source of adipocytes over time. The adipocyte precursors followed a pro-inflammatory trajectory characterized by elevated IL6 as WT mice aged. Strikingly, this pro-inflammatory program was already activated at 8 weeks in 3A-HET mice, months before development of obesity, suggesting that DNMT3A is required for proper adipocyte differentiation and prevents premature activation of a proinflammatory cascade. Differences in DNA methylation profiles between young and old WT and HET preadipocytes correlated closely with the precocious activation of the inflammatory cascade with reduced DNMT3A.

In obesity, adipose tissue inflammation is triggered by intrinsic signals including hypoxia, adipocyte death, and elevated lipid levels, which in turn exacerbate tissue dysfunction, lipotoxicity, inflammation, and metabolic disease (Reilly and Saltiel, 2017). Not only did loss of Dnmt3a alter adipocyte development, it also predisposed adipocytes towards a proinflammatory state prior to elevation of lipid levels. These results indicate that reduced DNMT3A impacts preadipocytes similarly to high fat diet-mediated obesity. Taken together, our study suggests that DNMT3A is essential for normal adipose tissue function over time, and DNMT3A may safeguard from inflammatory obesity in WT populations.

Given the known role of DNMT3A in regulating energy via the CNS, our data suggest a convergence with its activity in the adipose tissue. The constitutive reduced DNMT3A in TBRS patients creates a domino effect leading to the development of marked inflammatory obesity and metabolic disease. In future studies, it will be important to investigate how other tissues, such as the liver and the hematopoietic system, integrate into this cascade to contribute to obesity and its comorbidities. 


\section{Materials and Methods}

\section{Mouse models}

All mice were housed in AAALAC-accredited, specific-pathogen-free animal care facilities at Baylor College of Medicine, and all procedures were approved by the BCM Institutional Animal Care and Use Committee. Mice of both sexes were used unless stated otherwise, and experimental mice were separated by sex and housed with 4 mice per cage. All mice were immune-competent and healthy prior to the experiments described. Mice were bred and maintained at regular housing temperatures $\left(23^{\circ} \mathrm{C}\right)$ and $12-\mathrm{h}$ light/12-h dark cycle starting at 7:00 a.m. Animals had ad libitum access to water and chow diet. For physiological fasting experiments, mice were fasted from 7:00 pm to 7:00 a.m. To generate germline Dnmt3a heterozygous haploinsufficient mice we crossed $D n m t 3 a^{f / f l}$ mice previously obtained from the Beaudet laboratory (Challen et al., 2011) with E2a-cre mice obtained from Jackson labs. To generate adipocyte progenitor-specific depletion of DNMT3A, we also crossed the Dnmt3a $a^{\text {fl/fl }}$ to a Prx-Cre obtained from Jackson labs. We focused our analysis on males expressing the PrxCre in which transgene regulation has high fidelity. Accordingly, all comparisons, with knockout Cre models were done on male mice at 6-8 months of age.

\section{Cell culture and differentiation of preadipocytes}

3T3-L1 and BAC-C4 preadipocytes were kindly obtained from the laboratory of Mikhail Kolonnin. For differentiation assays cells were plated at $5 \times 10^{5}$ until confluent in $10 \%$ FBS high glucose DMEM. Following they were induced to differentiate, using the adipose-differentiation cocktail described below. For 3T3L1: cells were treated for 48 hours with induction media (25 $\mathrm{mM}$ indomethacin, $1 \mathrm{mM}$ dexamethasone, $0.5 \mathrm{mM}$ IBMX, $1 \mathrm{mM}$ insulin), next media was replaced and supplemented only with $1 \mathrm{mM}$ insulin for an additional 4 days until differentiation was complete and lipid droplets were formed. For BAC-C4: cells were treated for 48 hours with induction media (25 mM indomethacin, $1 \mathrm{mM}$ dexamethasone, $0.5 \mathrm{mM}$ IBMX, $0.5 \mathrm{mM}$ rosiglitazone, $1 \mathrm{mM}$ insulin). Following media was replaced to differentiation media (0.5 $\mathrm{mM}$ rosiglitazone $1 \mathrm{mM}$ insulin) for an additional 48 hours, next media was replaced and supplemented only with $1 \mathrm{mM}$ insulin for an additional 48 hours.

\section{Mouse phenotypic methods}




\section{Mouse body imaging}

For mouse body imaging of fat and lean tissues we used Dual energy x-ray absorptiometry (DEXA) for body composition and Bone Mineral Density at 6 months and 1-year old mice. To compare changes to bone growth and size we also used CT (3D X-Ray) Trifoil eXplore scanner.

\section{Comprehensive assessments of energy balance (CLAMS)}

For each CLMAS experiment we used WT and HET littermates at the age of 20-24 weeks. Both genders were used, 3 for each. Prior to recording of data mice were housed in the CLAMS feeder cages for 24 hours to familiarize themselves with the feeding system and to estimate food intake. Following mice were recorded for 4-5 days. All analyses were done by using CalR and data presented as significant are only parameters that were significant when accounted for body mass (Mina et al., 2018).

\section{Glucose and insulin tolerance test}

Mice were fasted for 12 hours, following they received IP injection of $20 \%$ glucose per $\mathrm{kg}$, for glucose tolerance test or $0.15 \mathrm{IU} / \mathrm{kg}$ insulin for insulin tolerance test. Blood samples are collected prior to and after the injection at time $0,15,30,60$, and 120 minutes via tail vein bleeding. Blood levels of glucose are measured based on using a glucometer. Fasting glucose or insulin levels were measured from plasma by glucose hexokinase assay by using Millipore rat/mouse insulin ELISA kit.

\section{Histopathology}

Animals were scarified, and tissues for downstream analysis were fixed in $4 \%$ (vol/vol) buffered formalin and embedded in paraffin. Sections were stained with hematoxylin and eosing using standard protocols. For measurement of adipocytes size and number, we used metamorph on $1 \mathrm{~mm}$ sections. Vacuole diameter size of 150 micrometer diameter was set as the upper limit, to avoid collection of broken cells. Quantification of Oil Red O droplets within liver section was done using imageJ

\section{Immunoblotting}

Cells or minced mouse tissue were lysed with 1x RIPA buffer supplemented with the Halt Protease and Phosphatase inhibitor cocktail (ThermoFisher) for 30 minutes. Following, protein concentration was quantified, and lysates were then boiled at 95C in 1x Laemmli (Bio-Rad) for 5 mins. The proteins were separated by SDS-PAGE on $4 \%-15 \%$ gradient gels (Bio-Rad), and 
transferred onto nitrocellulose membranes (Bio-Rad). After 1 hour of blocking in 3\% skim milk, membranes were incubated overnight with the following primary antibodies: anti- actin (Santa Cruz), anti-Dnmt3a (Cell signaling), anti-p-HSL (Cell Signaling), anti-STAT3 (Cell Signaling), anti-p-STAT3 (Cell Signaling). This was followed by secondary antibody incubation with antimouse or anti-rabbit horseradish peroxidase-conjugated secondary antibody (Santa Cruz), and imaging on the Bio-Rad ChemiDoc platform. Antibodies used: $\beta$-Actin Antibody (Cell Signaling, Cat\$4967S), phosphor-HSL(Ser660) (Cell Signaling. Cat \#4126S).

\section{Live cell staining and immunostaining}

For staining of lipid droplets, we used HCS LipidTOXTM following the manufacturer's instructions (Invitrogen). For live imaging of cells as described for fatty acids up-take we treated cells at day 5 of differentiation with BODIPYTM $(10 \mu \mathrm{M}) \mathrm{C}_{12}$ (4,4-Difluoro-5,7-Dimethyl-4-Bora-3a,4a-Diaza-sIndacene-3-Dodecanoic Acid) (Invitrogen). In addition, to measure cell growth cells were stained with Incucyte® NucLight Rapid Red Reagent for nuclear labeling following the manufacturer's instructions (Sartorius). Those experiments were monitored using Incucyte live cell analysis system. For immunostaining 7 days post differentiation, cells were plated on coverslips for 24 hours and following fixed in 4\% paraformaldehyde in PBS for $10 \mathrm{~min}$ at room temperature, rinsed with PBS, and permeabilized with $0.2 \%$ Triton X-100 in PBS for 5 min at room temperature. Blocking was done with normal goat serum for 1 hour. Following primary antibody phospho-HSL (Ser660) 1:100 (Cell signaling) was added for overnight incubation. All samples were then incubated in secondary antibody 1: 1000, Alexa Fluor 488 conjugate (Abcam), and mounted with ProLong Gold antifade reagent with DAPI (Invitrogen).

\section{Lipolysis assays}

For ex vivo lipolysis adipose tissue from inguinal, gonadal or brown adipose tissue were harvested from 6 months old WT and Dnmt3a-HET male mice following 6 hours fasting. Tissues were cut into small pieces of $10 \mathrm{mg}$ in duplicates and placed in Krebs buffer (114 mM NaCl, 4.7 mM KCl, 1.16 mM MgSO4, 1.2 mM KH2PO4, 2.5 mM CaCl2, 5 mM NaHCO3, 20 mM Hepes, $0.2 \%$ BSA, $2.5 \mathrm{mM}$ glucose). Next tissues were treated with isoproterenol (10 $\square \mathrm{nM})$ for $3 \square \mathrm{h}$ at $37 \square{ }^{\circ} \mathrm{C}$. Free-fatty-acids (FAA) and glycerol were measured using manufacturer's instructions (Sigma). To normalize for tissue size proteins were quantified for each sample. For 
measurement of protein content, the tissue was homogenized with RIPA cell lysis buffer (Milipore) and then lysate was quantified with Pierce BCA protein assay kit (Thermo Scientific).

\section{Stromal vascular cells (SVC) isolation and sort}

Adipose tissue from relevant depot harvested were minced in Krebs buffer (described above) supplemented with $1 \%$ bovine serum and $0.5 \mathrm{mg} / \mathrm{ml}$ collagenase I (Themo Scientific) and dispase $(2.4 \mathrm{U} / \mathrm{ml})$ (Sigma) following 45 minutes incubation at $37 \mathrm{C}$. Minced tissues were then washed with Hank's buffered salt solution (HBSS), supplemented with 10mM HEPES (GIBCO) and $2 \%$ heat-inactivated bovine serum (Corning). Following centrifugation steps (2000 rpm 5 minutes), the pellet was resuspended in red blood cell lysis buffer (155mM NH4CL, 12mM $\mathrm{NaHCO}$, $0.1 \mathrm{mM}$ EDTA) for 5 minutes. Following additional washing steps with HBSS, stromal vascular fraction was obtained and used for downstream analysis. For analysis of populations, the following antibodies were used: for removal of lineage positive cells: TER-119-PB (1:100), CD31-PB (1:100), CD45-PeCy7 (1:100). For isolation of preadipocytes, lineage negative cells were then selected for the expression of the following antibodies: Sca1- APC Cy7 (1:100), CD34-FITC (1:100), CD29-PE (1:200), IL6-APC (1:100) All monoclonal antibodies were from BD Biosciences or eBioscience. The LSRII cell analyzer was used for data acquisition, sorting was done using Arial. All data analysis was performed using the FlowJo software.

\section{Next generation sequencing approach and preparation}

All data relevant to this study has been deposited to GEO GSE164892

\section{Single cell RNA sequencing}

Sorted SVCs were used to make single cell RNA libraries using the 10x Genomics Chromium Platform. Libraries were sequence on NovaSeq to obtain minimum of 70,000 reads per cell to achieve $\sim 75 \%$ barcode saturation (Illumina). Filtering of samples were done by eliminating cells with mitochondrial contamination (<10\%), minimum of 500 genes per cell and minimum of 1000 mRNA molecules. Total number of cells used for final analysis ranged at 3000 total cells. From which: $\sim 1100$ cells were WT 8 weeks, $\sim 1100$ cells Dnmt3a-HET 8 weeks, 500 cells WT 1-year and $\sim 350$ cells Dnmt3a-HET 1 year.

\section{Single cell RNA processing}

All libraries were analyzed using Seruat pipeline (Butler et al., 2018). We integrated samples using scTransform with 3000 features (parameters: normalization method $=$ sct, dims= 1:20, nfeatures $=3000$ ). Next we regressed cell cycle and mitochondrial content. PCA and Shared 
nearest neighbors were performed using default Seruat functions with FindNeighbors and with parameters $\operatorname{dim}=1: 30$. For clustering we used the RunUMAP function from Seruat (paraments: $\operatorname{dim}=1: 30$, n.epochs $=500$, min.dist $=0.4)$. Clusters were identified using FindClusters (default). Clusters with low representation across all samples were removed $(<10 \%$ of population) leading to the removal of two clusters.

\section{Pseudotime algorithms}

To identify trajectories and cell lineages we used the following algorithms: destiny and slingshot. In order to identify the cell cluster to be used at the root of the trajectory analysis we performed destiny (diffusion component analysis). Following we used the DiffusionMap on the principle components as defined on the WT 8-weeks single cell RNAseq sample (number of PCA 1:20). Slingshot was used next to identify cellular relationships with setting of cluster 5 as the beginning of the node tree (parameters: start= cluster 5 , allow breaks $=$ false).

\section{Single cell differential gene expression}

We used the Seruat function FindAllMarkers (parameters test.use= DESeq2) to identify marker genes unique to each cluster when compared to all other clusters. To identify differential expression between genotypes or conditions we used FindMarkers (parameters: test.use= DESeq2). All plotting of differential of individual genes were done using either Seruat or scanpy default plotting setup (Challen et al., 2011).

\section{Knockout of Dnmt3a in preadipocytes cell lines}

Knockout of Dnmt3a was done using Crispr-CAS9 and dual guides with the previously described ribonucleoprotein (RNP) delivery method(Gundry et al., 2016). Guides were synthesized by T7- in vitro transcription according to the manufactures instructions (NEB) (Template for guide 1 and 2 in the table below). For genotyping the following primers were used:

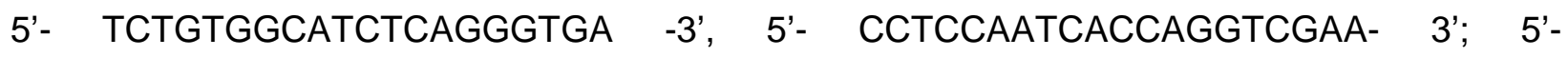
AGAGAGTGAGCACAGGCCAT-3'. Following validation of successful deletion, serial dilutions were done to isolate individual cell clones.

\section{RNA sequencing and analysis}

RNA was extracted using RNeasy micro kit (Qiagen) and quantified using Nanodrop. We prepared the Truseq stranded mRNA library using the manufacturer's instructions (Illumina). 
Libraries were sequenced using Nextseq 500 sequencer. Paired-end RNA-seq reads were mapped to mouse genome ( $\mathrm{mm} 10)$ using Hisat 2. Reads were quantified using either Feature Counts or by calculation of the Fragments Per Kilobase of exon per million fragments mapped (FPKM) values using Cufflinks 2.2.1 as defined per each dataset (Trapnell et al., 2012). Genes were defined as significant with $\mathrm{FPKM}>=10$, a fold change $>=2$ and with student $\mathrm{t}$-test $<=0.05$.

\section{Whole Genome Bisulfite Sequencing (WGBS) and Analysis}

Preadipocytes cells were enriched from white adipose tissue using magnetic depletion of CD45 and CD31. Following, we extracted DNA from cell pellets using DNeasy kit (Qiagen 69504). DNA was fragmented prior to bisulfite conversation using NEBNext fragmentase kit, following the manufacturer's protocol (NEB). We used $10 \mathrm{ng}$ DNA to prepare WGBS libraries with the Swift-whole genome bisulfite kit according to the manufacturer's instruction (Swift). Libraries were sequenced using an IlluminaNextseq 500 sequencer. Trimming of swift libraries was done using Trim Galore. Alignment and bisulfite conversation were done using Bisamrk (Challen et al., 2011). The WALT pipeline was used for duplicates removal using duplicate-remover as part of the WALT pipeline. We then used methcounts to calculate the methylation ratio of each CpG covered with at least 5 reads (Chen et al., 2016a).

\section{Analysis and definition of hypomethylated regions (HMRs)}

To identify HMRs in the adipose tissue WGBS data we used default settings. Briefly, a two-state hidden Markov model-based method was used for the HMR calling (Song et al., 2013). This model compares one state representing HMRs to highly methylated background from the same genomic DNA source. We then calculated the mean methylation of HMRs using the methylation ratio for each CpG (Coverage 5x per) as calculated above. To identify differentially methylated regions (DMRs) that changed in Dnmt3a-HET cells compared to WT, we pooled all HMRs from both genotypes, and HMRs that were within $200 \mathrm{bp}$ from each other were merged. Within those overlapping HMRs, we defined DMRs that differ between genotypes by an average of $30 \%$ for the ageing DMRs and 20\% for the DNMT3A dependent DMRs.

\section{Gene ontology and gene enrichment}

We used MsigDB and GSEA for gene enrichment analysis and hallmark pathway enrichment for RNAseq data and other genomic loci (Chen et al., 2013; Subramanian et al., 2005). For the 
correlation between DMRA and the scRNA expression data we first identified genes differential (average log2 fold) within groups and cellular states as defined in the results section using the FindMarkers function from Seruat version 3.9.9 (logFC threshold $=0$, minimum percentage $=0$, bonferroni $p$-value). Next, we ranked genes by their fold change value and used the fGSEA analysis.

\section{Differentially methylated regions definition and analysis}

Differentially methylated regions were initially defined on bases of the WT adipose methylation profile as either hypomethylated (methylation decreased $\geq 30 \%$ ) or hypermethylated (methylation gain $\geq 30 \%$ ) with age (hypo-DMRA and hyper-DMRA, respectively). For the purpose of methylation and gene expression correlations the association between the methylation status in those regions in HET adipose were further defined based on Fig. 6C. For HET adipose we defined: WT hypo-DMRA and HET 8 weeks hypomethylated by at least $19 \%$ (blue regions from Fig. 7C) or regions defined as WT hyper-DMRA and HET 8 weeks and 1year hypomethylated by at least 19\% (purple regions from Fig. 7G). 


\section{Author Contributions}

AT and MAG conceived the project, discussed and designed experiments. AT performed experiments and analysis with assistance of $C R, Y H H, A G G, S G, C H H, L Z, R R$ and ZG. XY and QT assisted with phosphor-STAT staining and analysis. ARC, SMH and MGK assisted with advice and critical review. MGK provided cell lines. JMR conducted most and supervised all the bioinformatic analysis in the manuscript. AT, JMR and MAG wrote the manuscript. All authors interpreted the results and edited the manuscript.

\section{Acknowledgments}

We thank C. Gillespie and K. Turner for critical review. We thank P. Saha, N. Putluri and C.S. Ward for their assistance and scientific advice as well as the Baylor College of Medicine Cores: Genomic and RNA Profiling, the Cytometry and Cell Sorting, Metabolomics, and Mouse Metabolism and Phenotyping. We thank the J Tadross, J Warner, B Mahler-Araujo and A VidalPuig from the Wellcome Trust-Medical Research Council Institute of Metabolic Science at the UK University of Cambridge for their assistance. This work was supported by NIH grants: HG006348, DK114356, HL130249, RR024574, CA183252, DK092883, AG036695, CA222736, CA125123, CPRIT-RP180672 and from the HHMI James H. Gilliam Fellowships.

\section{Declaration of Interests}

The authors declare no competing financial interests.

\section{Data availability}

All data relevant to this study has been deposited to GEO GSE164892 


\section{References}

Badman, M.K., and Flier, J.S. (2007). The adipocyte as an active participant in energy balance and metabolism. Gastroenterology 132, 2103-2115.

Balamurugan, K., and Sterneck, E. (2013). The many faces of C/EBPdelta and their relevance for inflammation and cancer. Int J Biol Sci 9, 917-933.

Butler, A., Hoffman, P., Smibert, P., Papalexi, E., and Satija, R. (2018). Integrating single-cell transcriptomic data across different conditions, technologies, and species. Nat Biotechnol 36, 411-420.

Challen, G.A., and Goodell, M.A. (2020). Clonal hematopoiesis: mechanisms driving dominance of stem cell clones. Blood 136, 1590-1598.

Challen, G.A., Sun, D., Jeong, M., Luo, M., Jelinek, J., Berg, J.S., Bock, C., Vasanthakumar, A., $\mathrm{Gu}, \mathrm{H}$., Xi, Y., et al. (2011). Dnmt3a is essential for hematopoietic stem cell differentiation. Nat Genet 44, 23-31.

Chen, E.Y., Tan, C.M., Kou, Y., Duan, Q., Wang, Z., Meirelles, G.V., Clark, N.R., and Ma'ayan, A. (2013). Enrichr: interactive and collaborative HTML5 gene list enrichment analysis tool. BMC Bioinformatics 14, 128.

Chen, H., Smith, A.D., and Chen, T. (2016a). WALT: fast and accurate read mapping for bisulfite sequencing. Bioinformatics 32, 3507-3509.

Chen, Y.S., Wu, R., Yang, X., Kou, S., MacDougald, O.A., Yu, L., Shi, H., and Xue, B. (2016b). Inhibiting DNA methylation switches adipogenesis to osteoblastogenesis by activating Wnt10a. Sci Rep 6, 25283.

Choquet, H., and Meyre, D. (2010). Genomic insights into early-onset obesity. Genome Med 2, 36.

Christian, D.L., Wu, D.Y., Martin, J.R., Moore, J.R., Liu, Y.R., Clemens, A.W., Nettles, S.A., Kirkland, N.M., Papouin, T., Hill, C.A., et al. (2020). DNMT3A Haploinsufficiency Results in Behavioral Deficits and Global Epigenomic Dysregulation Shared across Neurodevelopmental Disorders. Cell Rep 33, 108416.

Eguchi, J., Wang, X., Yu, S., Kershaw, E.E., Chiu, P.C., Dushay, J., Estall, J.L., Klein, U., Maratos-Flier, E., and Rosen, E.D. (2011). Transcriptional control of adipose lipid handling by IRF4. Cell Metab 13, 249-259.

Ertunc, M.E., and Hotamisligil, G.S. (2016). Lipid signaling and lipotoxicity in metaflammation: indications for metabolic disease pathogenesis and treatment. J Lipid Res 57, 2099-2114.

Friesen, M., Camahort, R., Lee, Y.K., Xia, F., Gerszten, R.E., Rhee, E.P., Deo, R.C., and Cowan, C.A. (2017). Activation of IRF1 in Human Adipocytes Leads to Phenotypes Associated with Metabolic Disease. Stem Cell Reports 8, 1164-1173.

Gundry, M.C., Brunetti, L., Lin, A., Mayle, A.E., Kitano, A., Wagner, D., Hsu, J.I., Hoegenauer, K.A., Rooney, C.M., Goodell, M.A., et al. (2016). Highly Efficient Genome Editing of Murine and Human Hematopoietic Progenitor Cells by CRISPR/Cas9. Cell Rep 17, 1453-1461. 
Jeong, M., Park, H.J., Celik, H., Ostrander, E.L., Reyes, J.M., Guzman, A., Rodriguez, B., Lei, Y., Lee, Y., Ding, L., et al. (2018). Loss of Dnmt3a Immortalizes Hematopoietic Stem Cells In Vivo. Cell Rep 23, 1-10.

Kohno, D., Lee, S., Harper, M.J., Kim, K.W., Sone, H., Sasaki, T., Kitamura, T., Fan, G., and Elmquist, J.K. (2014). Dnmt3a in Sim1 neurons is necessary for normal energy homeostasis. J Neurosci 34, 15288-15296.

Krueger, K.C., Costa, M.J., Du, H., and Feldman, B.J. (2014). Characterization of Cre recombinase activity for in vivo targeting of adipocyte precursor cells. Stem Cell Reports 3, 1147-1158.

LaPlant, Q., Vialou, V., Covington, H.E., 3rd, Dumitriu, D., Feng, J., Warren, B.L., Maze, I., Dietz, D.M., Watts, E.L., Iniguez, S.D., et al. (2010). Dnmt3a regulates emotional behavior and spine plasticity in the nucleus accumbens. Nat Neurosci 13,1137-1143.

Ley, T.J., Ding, L., Walter, M.J., McLellan, M.D., Lamprecht, T., Larson, D.E., Kandoth, C., Payton, J.E., Baty, J., Welch, J., et al. (2010). DNMT3A mutations in acute myeloid leukemia. N Engl J Med 363, 2424-2433.

Li, E., Bestor, T.H., and Jaenisch, R. (1992). Targeted mutation of the DNA methyltransferase gene results in embryonic lethality. Cell 69, 915-926.

Lim, J.Y., Duttke, S.H., Baker, T.S., Lee, J., Gambino, K.J., Venturini, N.J., Ho, J.S.Y., Zheng, S., Fstkchyan, Y.S., Pillai, V., et al. (2021). DNMT3A haploinsufficiency causes dichotomous DNA methylation defects at enhancers in mature human immune cells. J Exp Med 218.

Liu, Z., Gan, L., Zhou, Z., Jin, W., and Sun, C. (2015). SOCS3 promotes inflammation and apoptosis via inhibiting JAK2/STAT3 signaling pathway in 3T3-L1 adipocyte. Immunobiology 220, 947-953.

Logan, M., Martin, J.F., Nagy, A., Lobe, C., Olson, E.N., and Tabin, C.J. (2002). Expression of Cre Recombinase in the developing mouse limb bud driven by a Prxl enhancer. Genesis 33, 7780.

Ma, X., and Kang, S. (2019). Functional Implications of DNA Methylation in Adipose Biology. Diabetes 68, 871-878.

Merrick, D., Sakers, A., Irgebay, Z., Okada, C., Calvert, C., Morley, M.P., Percec, I., and Seale, $P$. (2019). Identification of a mesenchymal progenitor cell hierarchy in adipose tissue. Science 364.

Mina, A.I., LeClair, R.A., LeClair, K.B., Cohen, D.E., Lantier, L., and Banks, A.S. (2018). CalR: A Web-Based Analysis Tool for Indirect Calorimetry Experiments. Cell Metab 28, 656-666 e651.

Morris, M.J., Na, E.S., Autry, A.E., and Monteggia, L.M. (2016). Impact of DNMT1 and DNMT3a forebrain knockout on depressive- and anxiety like behavior in mice. Neurobiol Learn Mem 135, 139-145.

Okano, M., Bell, D.W., Haber, D.A., and Li, E. (1999). DNA methyltransferases Dnmt3a and Dnmt3b are essential for de novo methylation and mammalian development. Cell 99, 247-257. 
Reilly, S.M., and Saltiel, A.R. (2017). Adapting to obesity with adipose tissue inflammation. Nat Rev Endocrinol 13,633-643.

Rosen, E.D., and Spiegelman, B.M. (2014). What we talk about when we talk about fat. Cell 156, 20-44.

Schwalie, P.C., Dong, H., Zachara, M., Russeil, J., Alpern, D., Akchiche, N., Caprara, C., Sun, W., Schlaudraff, K.U., Soldati, G., et al. (2018). A stromal cell population that inhibits adipogenesis in mammalian fat depots. Nature 559, 103-108.

Song, Q., Decato, B., Hong, E.E., Zhou, M., Fang, F., Qu, J., Garvin, T., Kessler, M., Zhou, J., and Smith, A.D. (2013). A reference methylome database and analysis pipeline to facilitate integrative and comparative epigenomics. PLoS One 8 , e81148.

Subramanian, A., Tamayo, P., Mootha, V.K., Mukherjee, S., Ebert, B.L., Gillette, M.A., Paulovich, A., Pomeroy, S.L., Golub, T.R., Lander, E.S., et al. (2005). Gene set enrichment analysis: a knowledge-based approach for interpreting genome-wide expression profiles. Proc Natl Acad Sci U S A 102, 15545-15550.

Tatton-Brown, K., Seal, S., Ruark, E., Harmer, J., Ramsay, E., Del Vecchio Duarte, S., Zachariou, A., Hanks, S., O'Brien, E., Aksglaede, L., et al. (2014). Mutations in the DNA methyltransferase gene DNMT3A cause an overgrowth syndrome with intellectual disability. Nat Genet 46, 385-388.

Tatton-Brown, K., Zachariou, A., Loveday, C., Renwick, A., Mahamdallie, S., Aksglaede, L., Baralle, D., Barge-Schaapveld, D., Blyth, M., Bouma, M., et al. (2018). The Tatton-BrownRahman Syndrome: A clinical study of 55 individuals with de novo constitutive DNMT3A variants. Wellcome Open Res 3, 46.

Tchkonia, T., Morbeck, D.E., Von Zglinicki, T., Van Deursen, J., Lustgarten, J., Scrable, H., Khosla, S., Jensen, M.D., and Kirkland, J.L. (2010). Fat tissue, aging, and cellular senescence. Aging Cell 9, 667-684.

Tovy, A., Reyes, J.M., Gundry, M.C., Brunetti, L., Lee-Six, H., Petljak, M., Park, H.J., Guzman, A.G., Rosas, C., Jeffries, A.R., et al. (2020). Tissue-Biased Expansion of DNMT3A-Mutant Clones in a Mosaic Individual Is Associated with Conserved Epigenetic Erosion. Cell Stem Cell 27, 326-335 e324.

Tovy, A., Rosas, C., Gaikwad, A.S., Medrano, G., Zhang, L., Reyes, J.M., Huang, Y.H., Arakawa, T., Kurtz, K., Conneely, S.E., et al. (2021). Perturbed hematopoiesis in individuals with germline DNMT3A overgrowth Tatton-Brown-Rahman syndrome. Haematologica.

Trapnell, C., Roberts, A., Goff, L., Pertea, G., Kim, D., Kelley, D.R., Pimentel, H., Salzberg, S.L., Rinn, J.L., and Pachter, L. (2012). Differential gene and transcript expression analysis of RNAseq experiments with TopHat and Cufflinks. Nat Protoc 7, 562-578.

Yang, X., Wu, R., Shan, W., Yu, L., Xue, B., and Shi, H. (2016). DNA Methylation Biphasically Regulates 3T3-L1 Preadipocyte Differentiation. Mol Endocrinol 30, 677-687. 
You, D., Nilsson, E., Tenen, D.E., Lyubetskaya, A., Lo, J.C., Jiang, R., Deng, J., Dawes, B.A., Vaag, A., Ling, C., et al. (2017). Dnmt3a is an epigenetic mediator of adipose insulin resistance. Elife 6.

Zhang, Y., Proenca, R., Maffei, M., Barone, M., Leopold, L., and Friedman, J.M. (1994). Positional cloning of the mouse obese gene and its human homologue. Nature 372, 425-432. 


\section{FIGURE LEGENDS}

\section{Fig 1: Dnmt3a-HET mice become overweight and develop obesity}

A. Weight of male Dnmt3a-HET (HET, $n=14)$ and WT $(n=12)$ littermates on regular chow. * $\mathrm{p}=0.03,{ }^{* *}<\mathrm{p}=0.001$ as determined by 2 way ANOVA.

B. Distribution of weight from HET and WT mice females and males at 1-year of age. Dashed lines indicate 3 standard deviations (SD) above the mean of the WT.

C. Representative display of D3A-HET and WT mice at 1 year

D. Left: D3A-HET and WT mice at 1 year. Right: dual energy X-ray absorptiometry (DEXA) analysis of 6-month-old WT and HET mice. Top: Representative image displaying lean (brown color) and fat (grey) tissues.

E. Quantification of fat and lean mass $(n=10-14)$ from DEXA. Statistical analysis 1way ANOVA

F. Representative H\&E staining of gonadal white adipose tissue and BAT from 1-year-old mice.

G. Morphometric analysis of gWAT and BAT adipocyte diameter. ${ }^{* *} \mathrm{p}<0.001$ by 1 way ANOVA

H. Representative staining of liver tissue from 1-year-old WT and 3A-HET littermates. Top: H\&E staining. Bottom: Oil Red O lysochrome diazo dye.

I. Quantification of total red droplets from Oil Red O-stained livers ( $n=3$ per group). Statistical analysis 1way ANOVA

Fig 2: Dnmt3a-HET mice display increased feeding and develop glucose and insulin intolerance

A. Average hourly food consumption for WT ( 3 males and 3 females) and HET mice (3 males and 3 females) as averaged for data collected over 5 days. Statical analysis ACNOVA

B. Energy balance calculated from calories consumed versus calories expended. For the mice as in a.

C. Levels of fasting glucose and insulin of WT and HET mice at 2 months of age. All data are expressed as mean \pm SEM.

D. Levels of fasting glucose and insulin of WT and HET mice at 6 months of age. All data are expressed as mean \pm SEM. 
E. Glucose tolerance test at 6 months of age $(n=10)$. Mice were fasted for 12 hours prior to beginning the test. All data are expressed as mean \pm SEM. ${ }^{*} p<0.05$, ${ }^{* *} p<0.01,{ }^{* \star *} p<$ 0.0001 by 2 way ANOVA.

F. Insulin tolerance test at 10 months of age $(n=10)$. Mice were fasted for 12 hours prior to beginning the test. All data are expressed as mean \pm SEM. ${ }^{*} p<0.05$, ${ }^{* *} p<0.01,{ }^{* \star *} p<$ 0.001 by 2 way ANOVA.

Fig 3: Dnmt3a-HET adipose tissue contains more stem cell-like preadipocytes at the expense of committed preadipocytes

A. Scheme of single cell RNA sequencing from stromal vascular cells (SVC) of WT and 3AHET adipose tissue.

B. Uniform Manifold Approximation and projection (UMAP) clustering of pooled preadipocyte cells from WT and 3A-HET at 8 weeks and at 1-year of age.

C. Top hallmark pathways of marker genes for each cluster shown as a heat map. Marker genes were enriched in a cluster compared to all other clusters.

D. Expression levels of cluster-defining marker genes depicted by violin plots. The y-axis marks the normalized log-scale of average read counts.

E. Top: UMAP representation of the stem cell marker Dpp4 and the committed preadipocyte marker Fabp4 across all clusters. Bottom: definition of cellular states for the clusters based on the levels of Dpp4 and Fabp4. Stem and progenitor cells had the highest Dpp4 with no Fabp4. Primed preadipocytes expressed both Dpp4 and Fabp4, and committed preadipocytes expressed mainly Fabp4.

F. Quantification of the denoted cluster proportions at 8 weeks $(8 \mathrm{w})$ and 1 year $(1 \mathrm{y}) .{ }^{*} \mathrm{p}<$ $0.05,{ }^{* *} p<0.01,{ }^{* * *} p<0.001$ by Fisher exact test.

G. Quantification of preadipocytes in stromal vascular cells (SVC) cells by flow cytometry using the following strategy: cells were selected as lineage negative (lacking Cd45, Cd31, and Ter119) and positively expressing Cd34, Sca1 and Cd29 cell in WT and 3AHET stromal vascular cells (SVCs) at 8 weeks and at 1 -year of age. ${ }^{*} p<0.05,{ }^{* *} p<0.01$ by Student's- t- test

Fig 4: Pseudotime diffusion analysis shows that loss of Dnmt3a leads to increase in proinflammatory cellular trajectory 
A. For each of the clusters as identified in 8-week-old WT mice preadipocytes we calculated diffusion score. The lowest diffusion value indicates that cells within the cluster display a more naïve stem-like gene expression profile.

B. Cell trajectories inferred by slingshot analysis overlaid on UMAP data (curved lines).

C. Expression levels of inflammatory marker genes that define clusters 1, 3 and 5, as depicted by violin plots. The y-axis marks the normalized log-scale of average read counts.

D. UMAP representation of the identified cell sates from B for cells from 8-week and 1-year old mice.

E. Gene enrichment analysis (MsigDB) based on differential gene expression as calculated from the single cell RNA seq data. For each of the specified groups we ranked genes by average log 2 fold change within state A or B and separated by genotype. Color indicates relative contribution of WT (blue) or HET (red) cells. Size of circle denotes bonferroni log transformed 10 adjusted $p$-value.

F. IL6 protein levels in permeabilizated SVCs that lack CD45 marker by flow cytometry. Left: representative quantification of 116 positive cells. Bottom: quantification of II6 positive cells as percentage of CD45-negative cells in adipocytes from 1-year old mice.

\section{Fig 5: DNMT3A regulates preadipocyte proliferation and differentiation}

A. Western blot probed with DNMT3A antibody after CRISPR KO in BAC-C4. Three clones of each are shown.

B. Proliferation of three WT and KO clones of BAC-C4 cells; assay based on water-soluble tetrazolium (MTT).

C. Representative staining of lipid droplets (LipidTOX (green)) following 6 days of differentiation of BAC-C4 control and KO cells. Scale $2 \mathrm{~mm}$.

D. Incorporation of fluorescent fatty acid (BODIPY FL $\mathrm{C}_{12}$ (green)) relative to red nuclear fluorescent staining quantified during live cell imaging at day 5 of differentiation. Statistical analysis 2 way ANOVA. ${ }^{* * *} p<0.0001$

E. Quantification of green, fluorescent fatty acid in control and $\mathrm{KO}$ cells following treatment with isoproterenol. Data shown is relative to the abundance of green fluorescence at baseline. Statistical analysis 2 way ANOVA. ${ }^{* * *} p<0.0001$

F. Representative immunostaining of cells with the indicated antibodies. Cells were differentiated for 5 days and then treated with isoproterenol for 24 hours 
G. RNAseq analysis at baseline (average of 3 clones from each genotype, non-treated, NT), at day 6 of differentiation (DIFF, 2 clones each genotype), and following 24 hours treatment of cells after 5 days of differentiation with isoproterenol (ISO, 2 clones each). Left panel: For genes defined as upregulated in BAC-control compared to KO following ISO, we plotted log transformed mean expression for all conditions. Right panel: Heatmap of MsigDB enrichment of genes upregulated in BAC-control (fold $>2, p<=0.05$ ) compared to BAC-KO (Fold $>2, \mathrm{p}<=0.005$ ).

$H$. Using RNAseq data from (E): Left panel: log transformed mean expression for all conditions for genes defined as upregulated in BAC- KO compared to control following ISO. Right panel: Heatmap of MsigDB enrichment of genes upregulated in BAC-KO (fold $>2, p<=0.05$ ) compared to BAC-Control (Fold $>2, p<=0.005$ ).

I. Heatmap of top 5 genes differentially expressed between control and $\mathrm{KO}$ and annotated to the hallmarks adipogenesis or Epithelial to mesenchymal transition (EMT) and TNFa signaling pathways.

Fig 6: DNMT3A regulates lipolysis and inflammatory gene pathways

A. Overlap of normalized enrichment score (NES) of GSEA hallmark pathway overlap between the scRNAseq data for the primed preadipocytes and BAC RNAseq at the nontreated (NT) state. For each dataset we performed GSEA analysis and calculated NES score. Positive values are pathways enriched for WT adipose and BAC control cells. Negative values are pathways enriched for HET adipose and BAC KO cells.

B. Overlap of normalized enrichment score (NES) of GSEA hallmark pathway overlap between the scRNAseq data for the committed preadipocytes and BAC RNAseq at day 6 of induced differentiation. For each dataset we performed GSEA analysis and calculated NES score. Positive values are pathways enriched for WT adipose and BAC control cells. Negative values are pathways enriched for HET adipose and BAC KO cells.

C. Quantification of free fatty acid in the plasma of 12 hours fasted mice.

D. Quantification of glycerol in the plasma of 12 hours fasted mice.

E. Quantification of free fatty acid release from gonadal fat (gWAT) from WT and 3A-HE mice fat was isolated from mice fasted for 6 hours and treated with $1 \mu \mathrm{m}$ isoproterenol for $3 \mathrm{~h}$.

F. Quantification of glycerol at the end of the experiments described in e. 
Fig 7: Loss of Dnmt3a in inguinal subcutaneous adipose progenitors leads to fat expansion

A. Weight of male Prx-Cre Dnmt3a mice (PRX-D3A) mice at 8 months of age in compared to controls (Dnmt3a $\left.{ }^{\text {floxlflox }}\right)(n=8-10)$. Student T-test was done for statistical analysis.

B. Dual energy X-ray absorptiometry (DEXA) analysis of PRX-D3A mice compared to control mice. Left: Quantification of fat mass $(n=10-12)$ from DEXA. Statistical analysis 1way ANOVA. Right: Representative image from DEXA.

C. Plasma leptin from Prx-Cre Dnmt3a mice compared to controls.

D. Quantification of preadipocytes in stromal vascular cells (SVC) cells from subcutaneous (Prx-Cre is expressed) and visceral (Prx-Cre not expressed) by flow cytometry using the following strategy: cells were selected as lineage negative (lacking Cd45, Cd31, and Ter119) and positively expressing Cd34, Sca1 and Cd29 cell in Prx-Cre Dnmt3a mice compared to control. 2-Way Anova was done for statistical analysis.

E. Quantification of free fatty acids (FAA) release from subcutaneous fat of Prx-Cre Dnmt3a mice compared to control. Fat pad was isolated from mice fasted for 12 hours and treated with $1 \mu \mathrm{m}$ isoproterenol for $3 \mathrm{~h}$.

Fig 8: Loss of Dnmt3a alters DNA methylation landscape during adipocyte development and ageing
A. Schematic for strategy to define regions hypomethylated with age (WT hypo-DMRA).
B. Violin plot of mean methylation for WT hypo-DMRA and their mean methylation in 3A- HET.
C. For the regions defined as WT hypo-DMRA we subtracted the methylation value for the same loci in 8 weeks HET cells. In this histogram representation we identified two peaks: in blue are regions that are hypomethylated in HET cells (20\% difference or greater) and in grey regions which share similar levels in methylation in WT ad HET cells and determined as unchanged regions.
D. Mean methylation $+/ 11 \mathrm{~Kb}$ from the DMR for regions defined as WT DMRA-hypo.
E. Schematic of definition of regions hypermethylated in WT ageing (WT hyper-DMRA). 
F. Violin plot of mean methylation for WT hyper-DMRA in HET cells (8 weeks and 1 year).

G. Scatter plot of methylation levels for WT hyper-DMRA and the same loci in HET at 8 weeks and at 1-year. Quantification of the correlation between the regions that remain hypomethylated (20\% or larger) in HET cells in 8 weeks and in 1-year (purple) indicates high probability score of them being hypomethylated in HET cells regardless of age.

Fig 9: Loss of DNA methylation in HET cells is associated with activation of the proinflammatory cell state $B$

A. Illustration of the cell clusters used for the gene enrichment analysis based on the clusters and cellular states identified in Fig 5.

B. Displayed is the enrichment score and the Bonferroni adjusted p-value for genes associated with WT hypo and HET hypo DMRA based on their enrichment within cell cluster groups and cellular sates as described in A. Bottom: Negative normalized enrichment score (NES) indicates more contribution of HET cells to the genes associated with the WT hypo DMRA regions, while positive NES indicates more contribution of WT cells to the genes associated the described regions in DMRA and in unchanged regions.

C. Displayed is the enrichment score and the Bonferroni adjusted p-value for genes associated with WT hyper and HET (8-weeks and 1-year) hypo DMRA based on their enrichment within cell cluster groups and cellular sates as described in A. Negative normalized enticement score (NES) indicates more contribution of HET cells to the genes associated with the WT hyper DMRA regions, while positive NES indicates more contribution of WT cells to the genes associated the described regions.

D. For regions defined as WT hyper-DMRA and HET 8 weeks and 1-year hypomethylated (purple regions from Fig. 6G) we performed gene enrichment analysis based on the cell clusters groups and cellular sates illustrated in A. Displayed is the enrichment score and the Bonferroni adjusted p-value in WT and HET cells.

E. DNA methylation at the F3, Dusp1 and Pdgfrb loci in WT (black) and HET (red) cells at 8 weeks and at 1-year. Highlighted are the regions which gain methylation in WT cells but lose methylation in the HET. 
bioRxiv preprint doi: https://doi.org/10.1101/2021.09.13.460063; this version posted September 15, 2021. The copyright holder for this preprin

(which was not certified by peer review) is the author/funder, has granted bioRxiv a license to display the preprint in perpetuity. It is $\mathrm{m}$ \&de 1

A

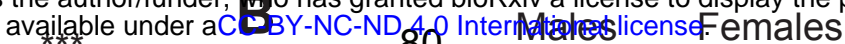
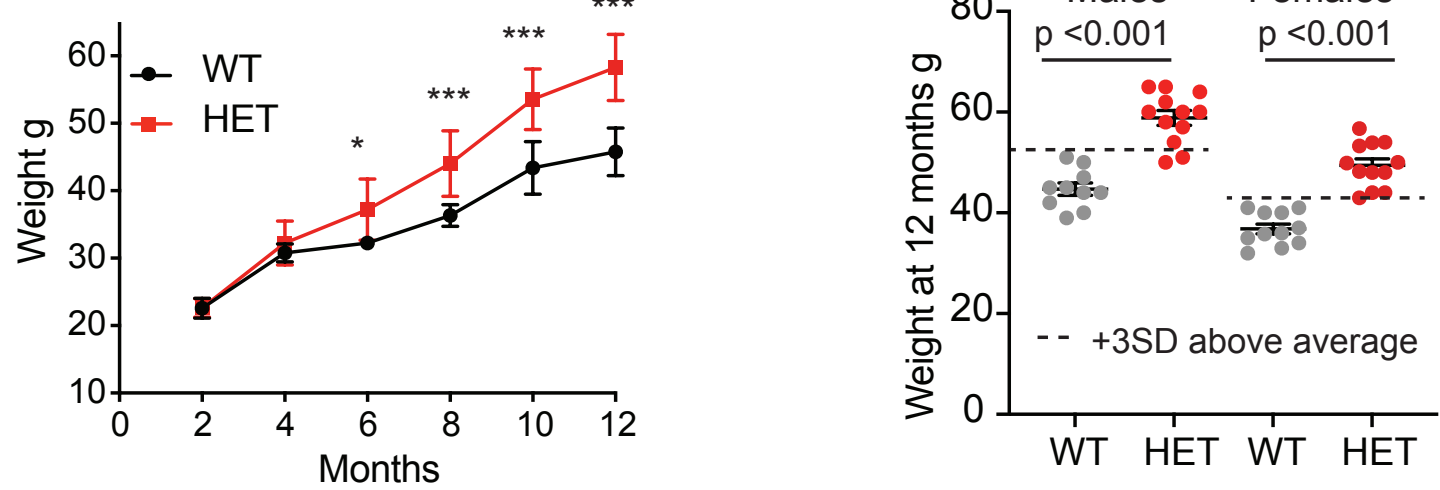

C

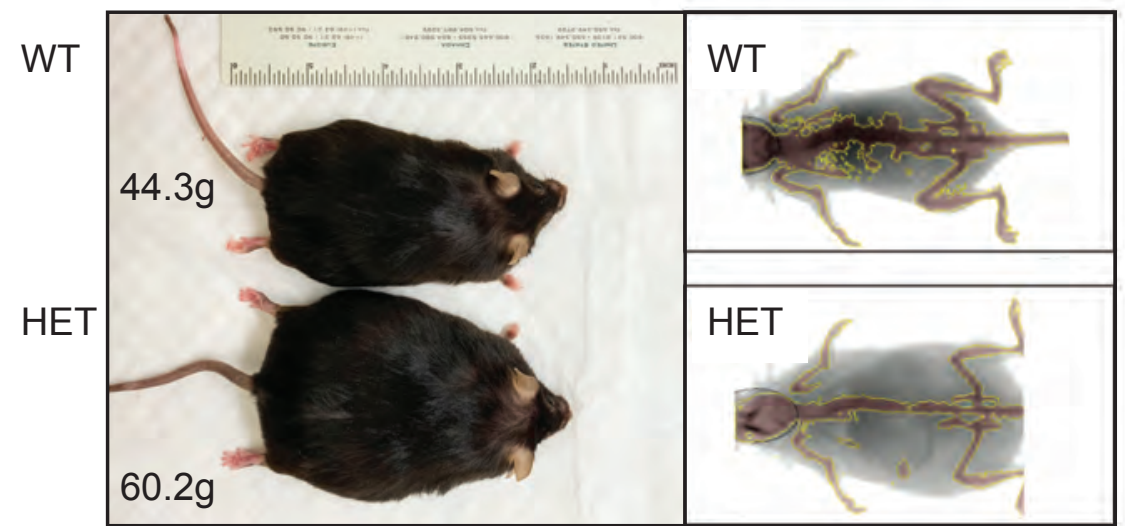

D

$\mathbf{E}$

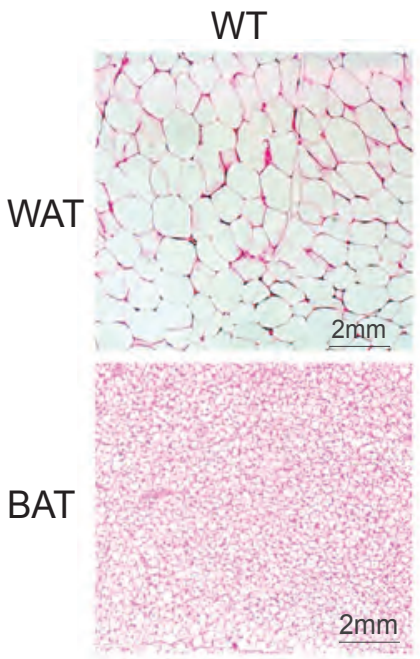

G
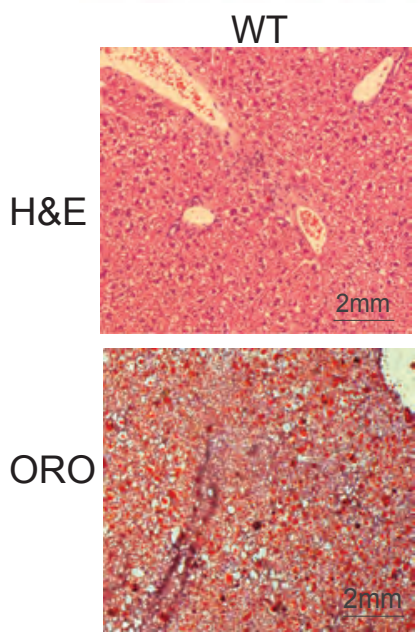

HET

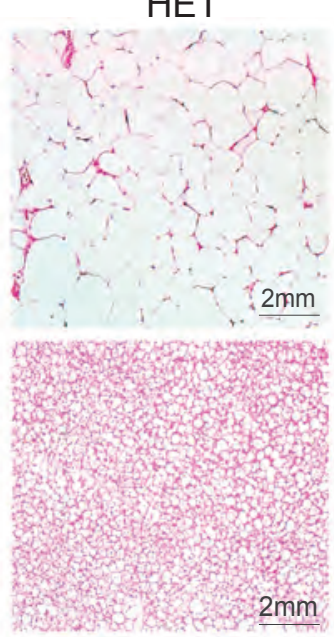

HET
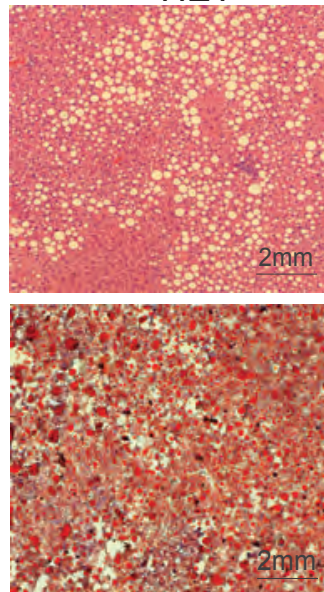

F
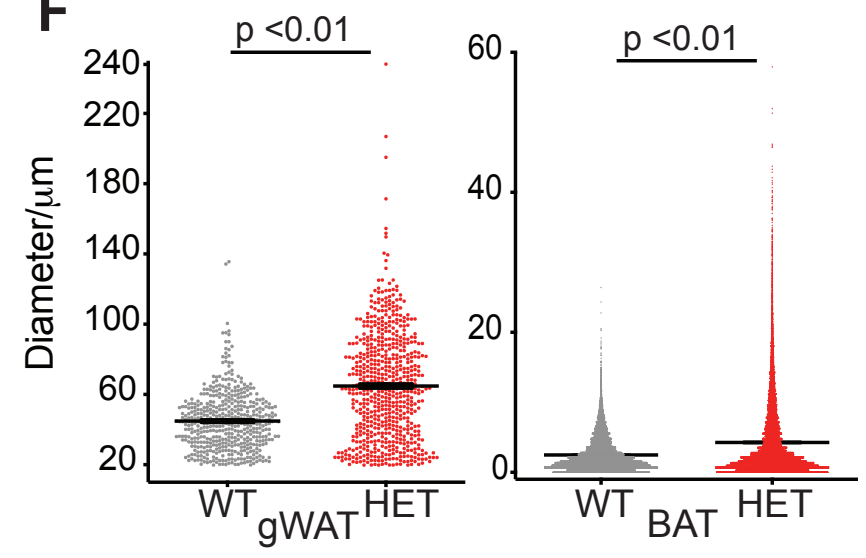

H

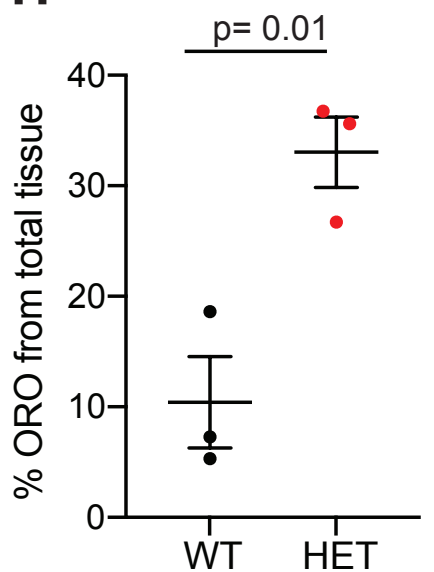


A

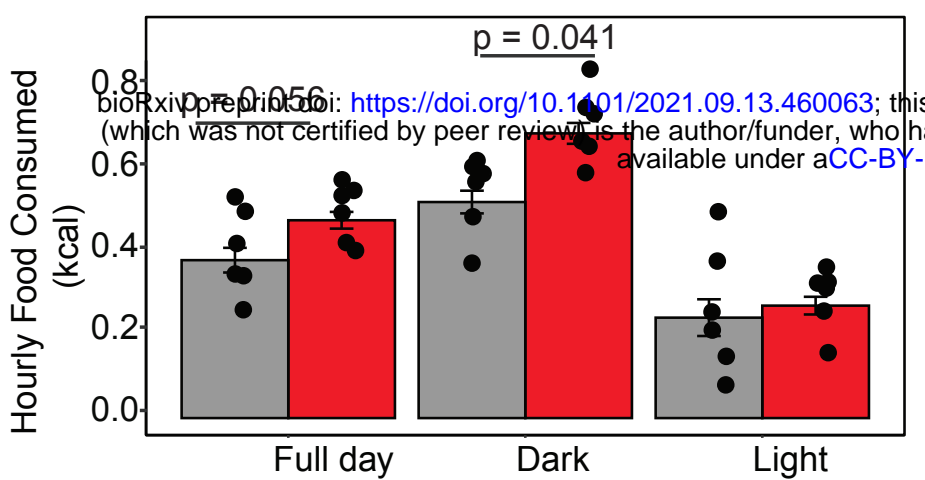

C

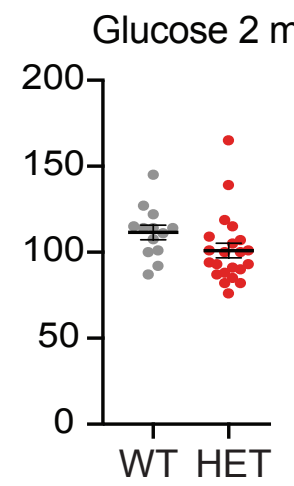

E

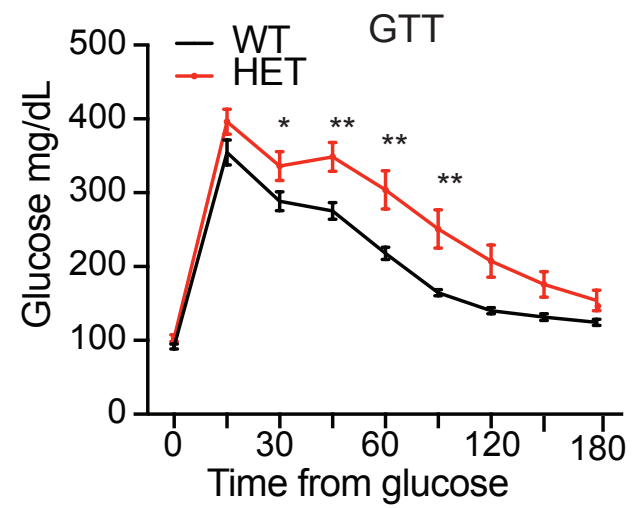

B

Figure 2

产

$\underline{p=0.03}$

ion posted September 15, 2021. The copyright holder for this preprint Ganted bloRxipa Ticense to display the preprt in perpetuity. It is made

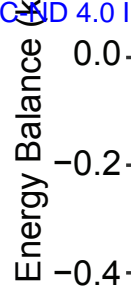

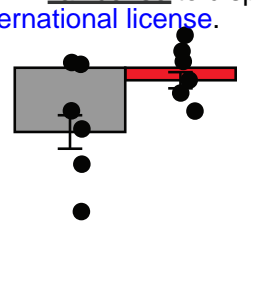

Full day

Dark

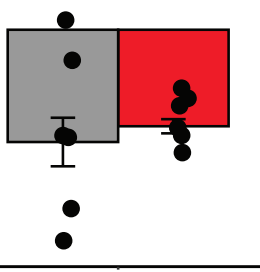

Light

D Glucose 6 mon Insulin 6 mon
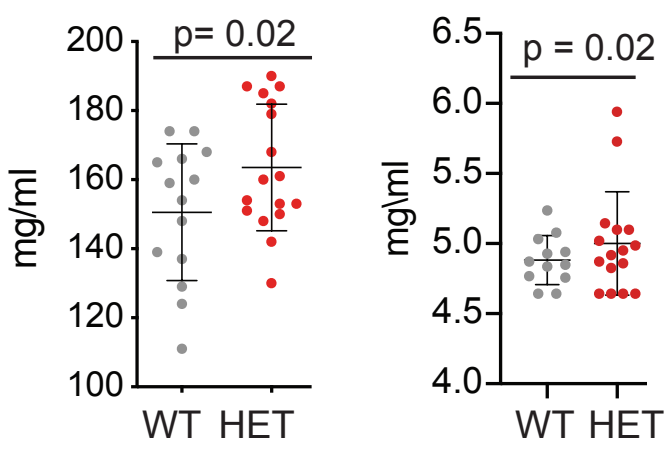

$\mathbf{F}$

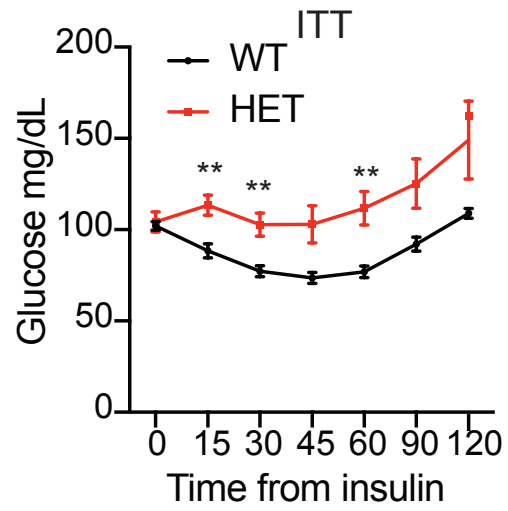




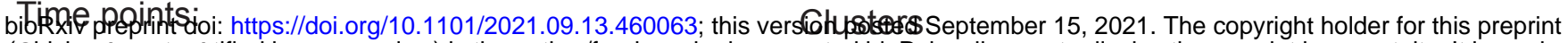

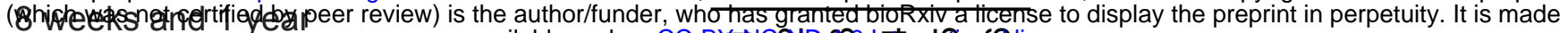

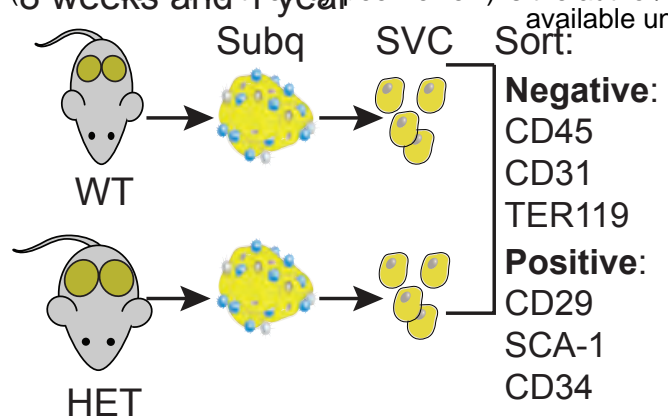

B
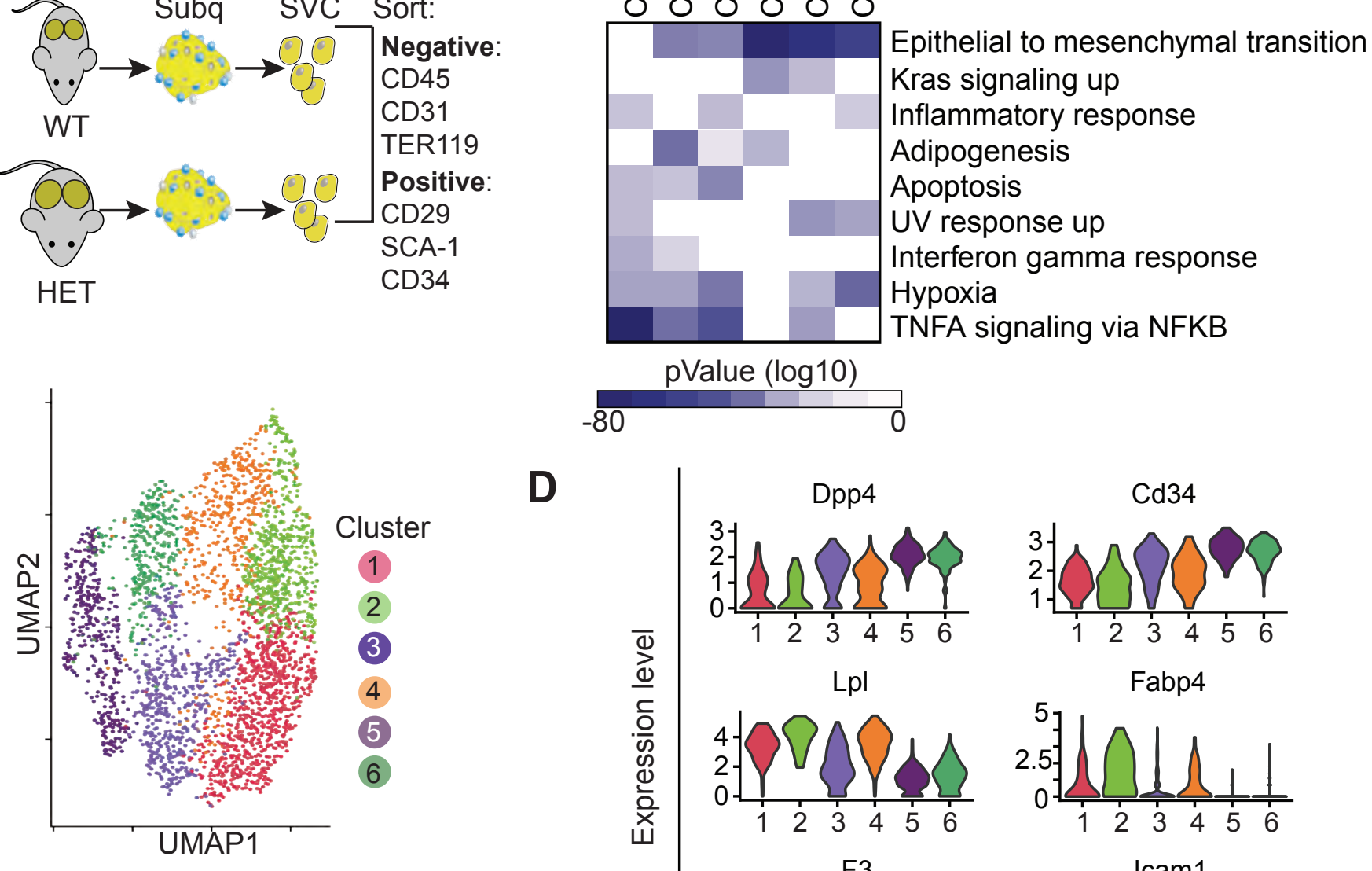

E
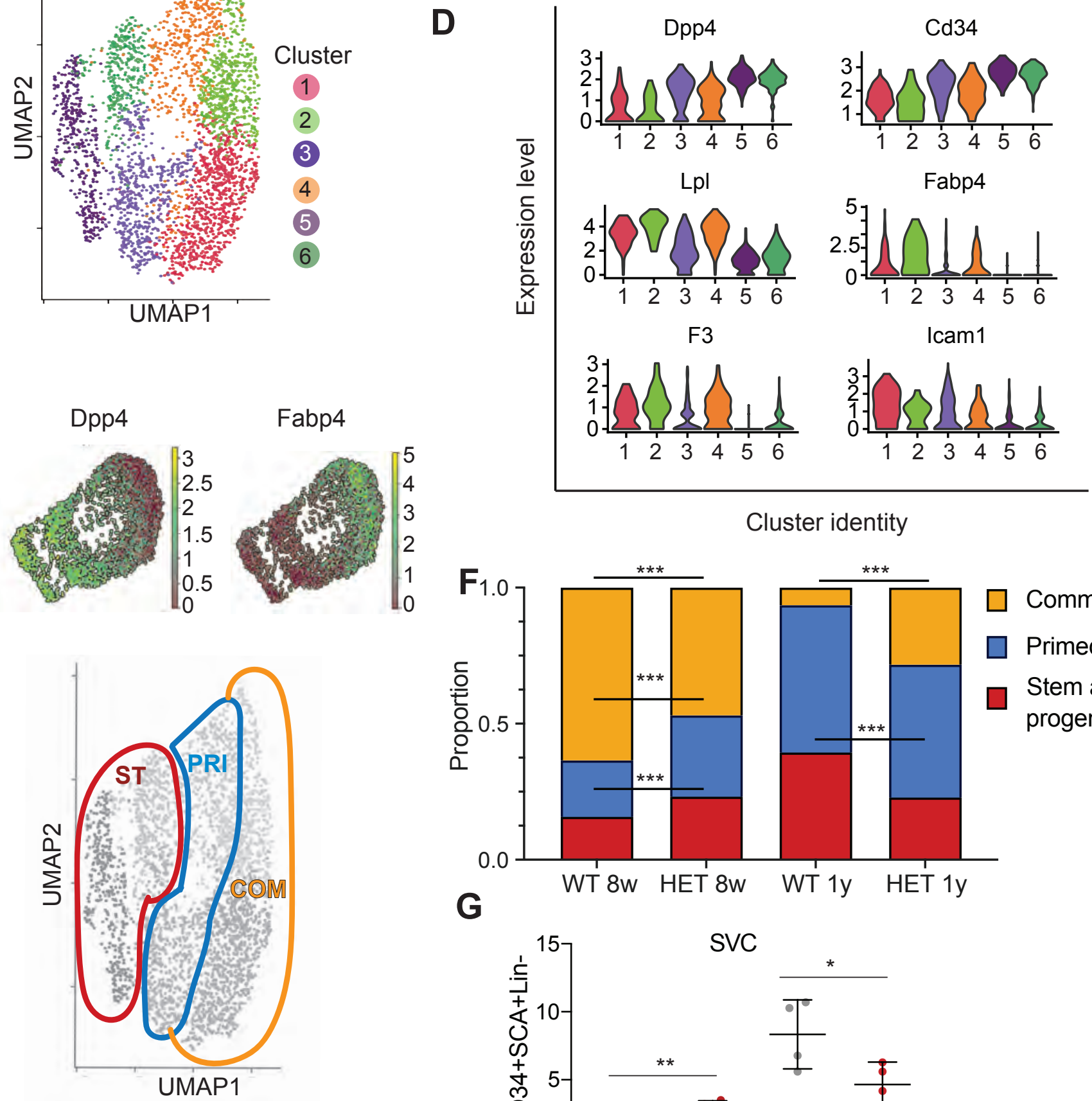

ST- Stem and progenitors

PRI- Primed preadipocytes

COM- Committed preadipocytes

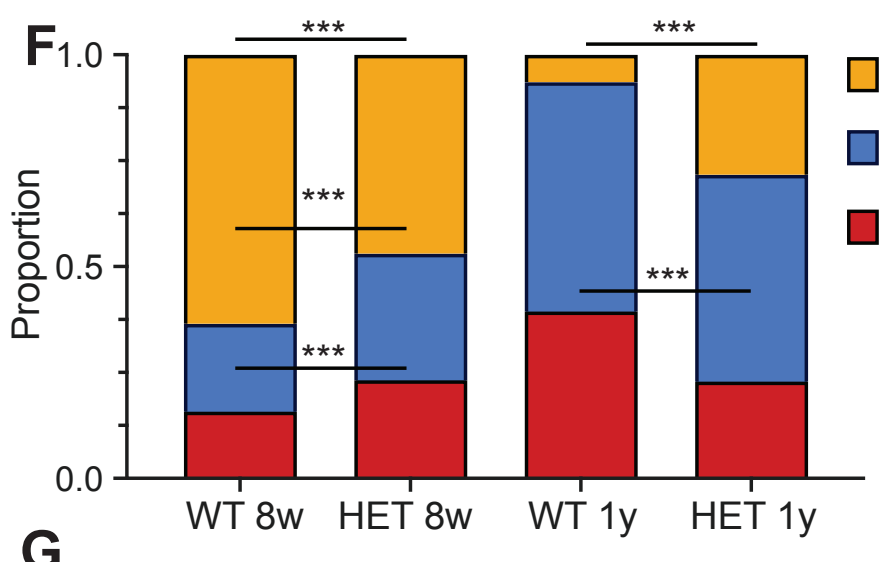

Committed

Primed

Stem and

progenitors

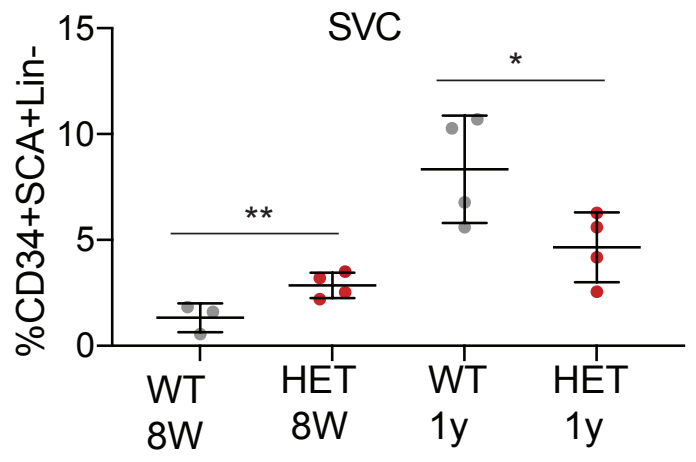


bioRxiv preprint doi: https://doi.org/10.1101/2021.09.13.460063; this version posted September 15, 2021. The copyright holder for this preprint

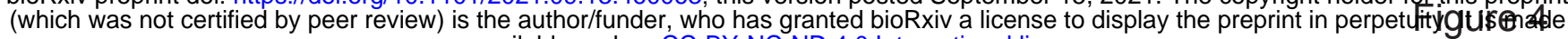
available under aCC-BY-NC-ND 4.0 International license.

A

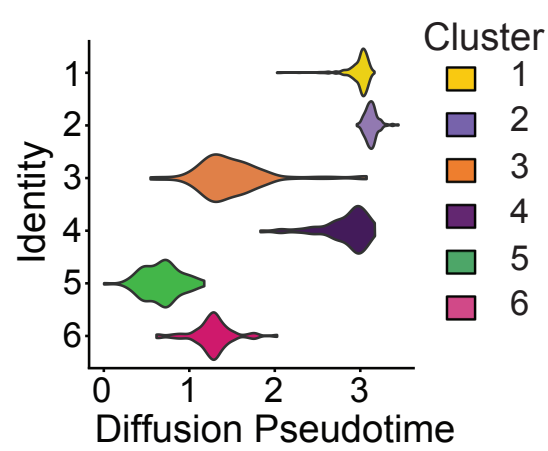

D
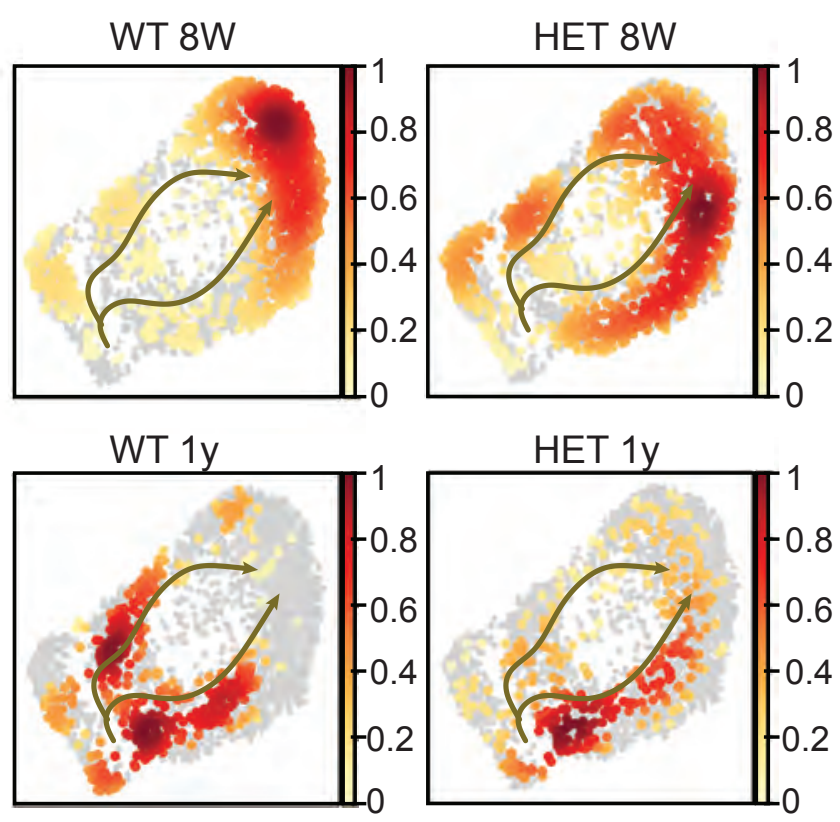

HET 1y

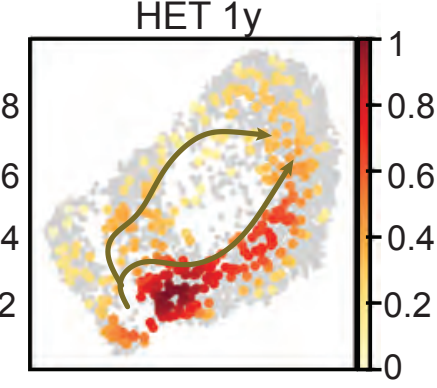

$\mathbf{F}$

IL6 in adipocytes
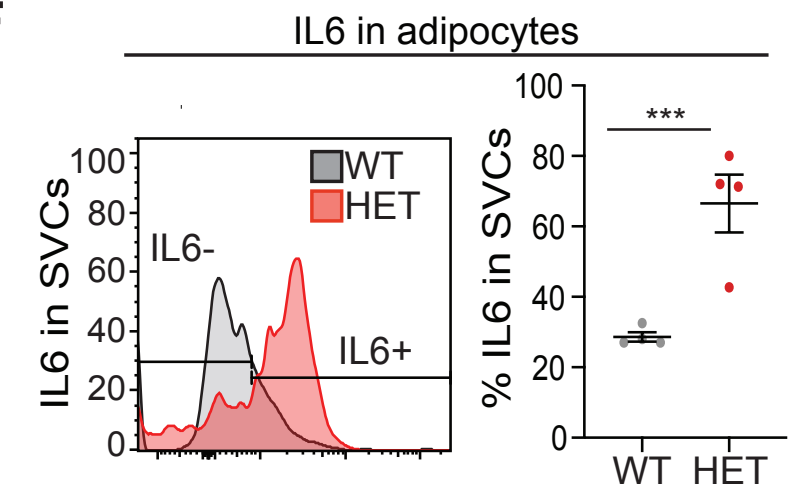

B

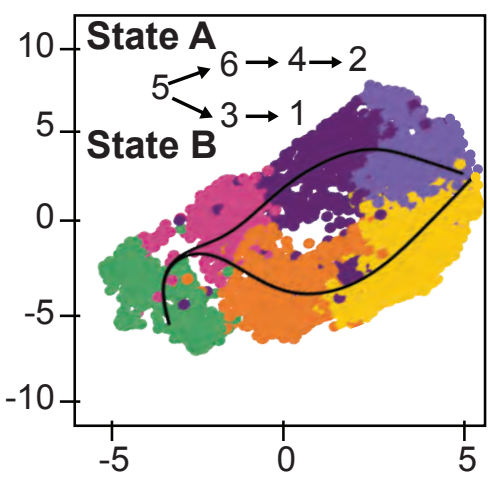

E
C

Cluster

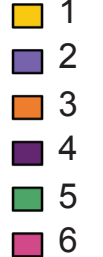

II6

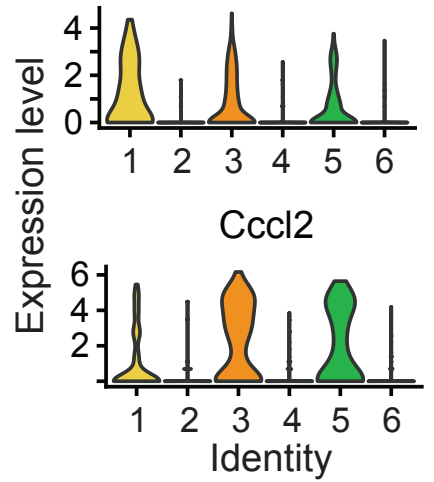


A

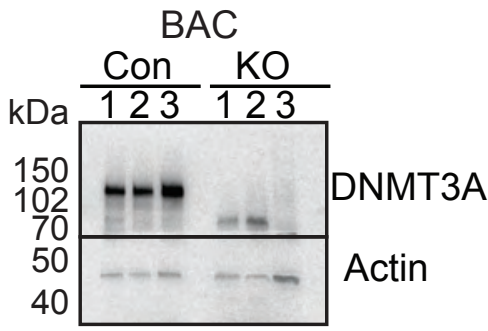

D
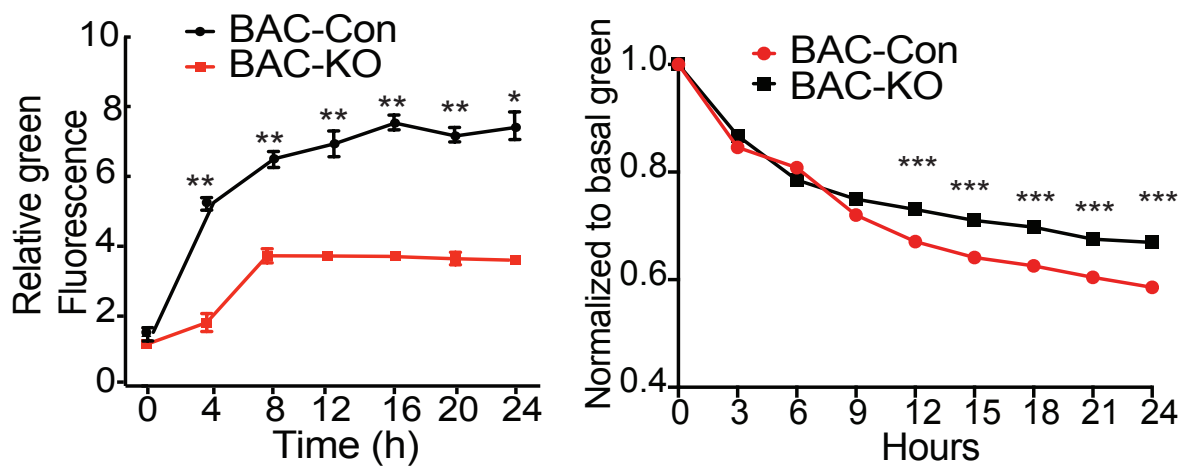

G

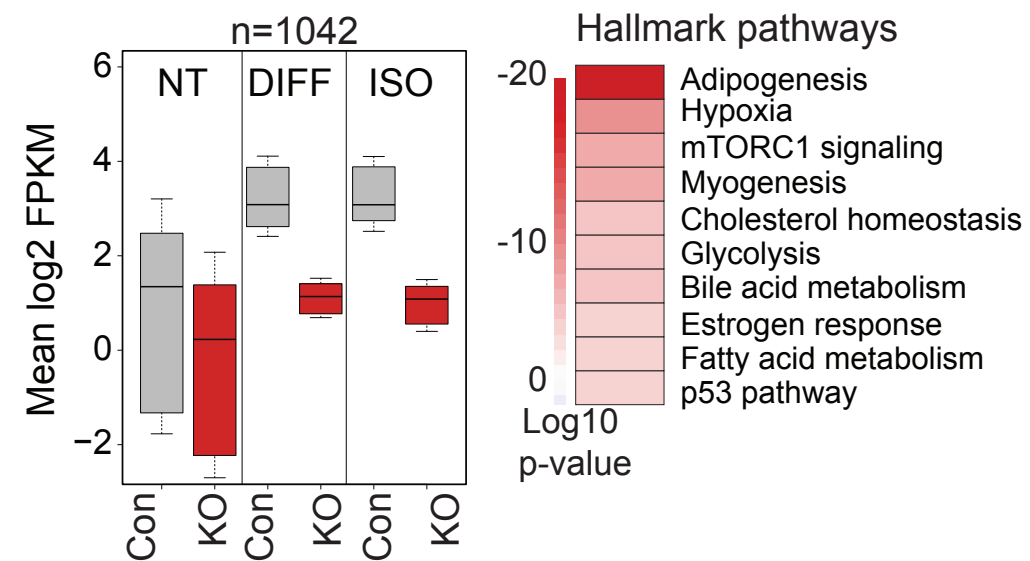

H

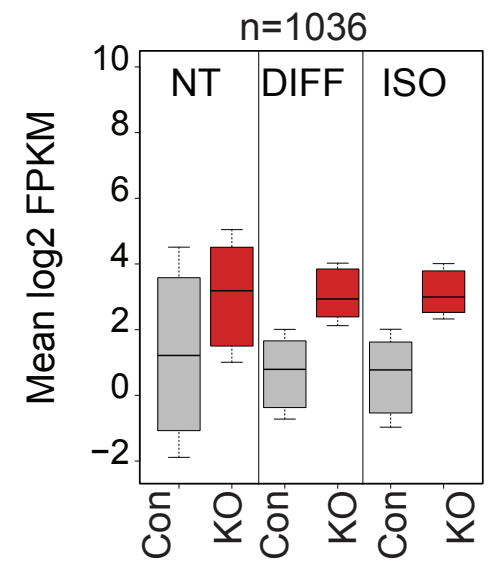

B

E
Hallmark pathways

$-20 \quad$ Epithelial to mesenchymal

TNFa signaling via NFKB

Allograft rejection

Estrogen response

Apoptosis

IFN-gamma reposnse

p53 pathway

UV response

0

IL2 STAT5 signaling

Log10

p-value
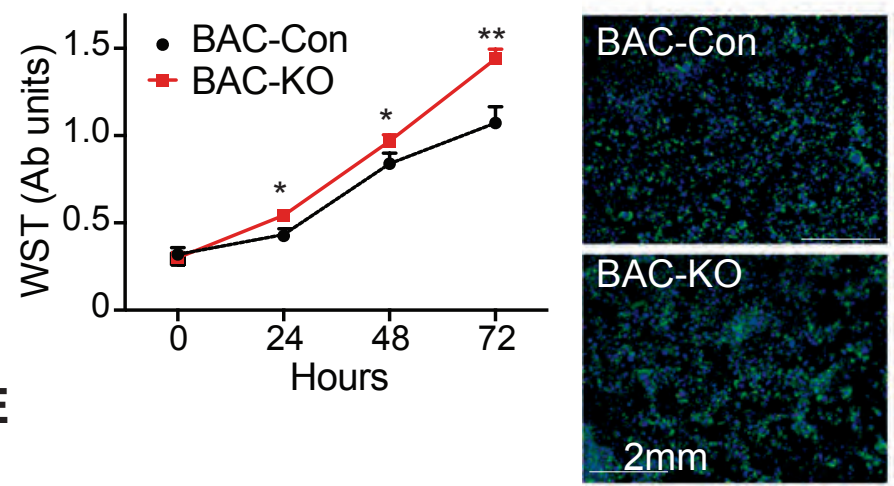

Color index: LipidTOX, DAPI

F
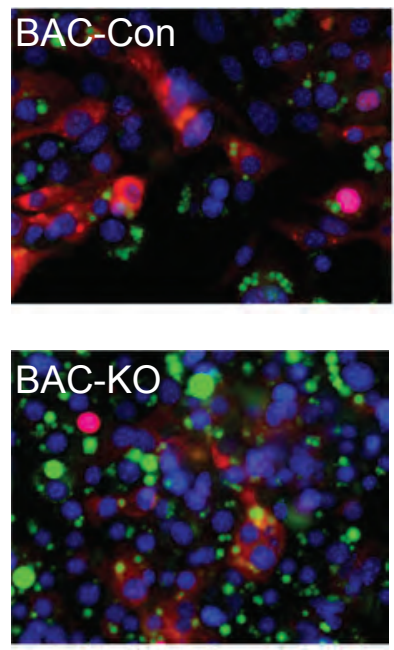

Color index:

LipidTOX, pHSL DAPI

I

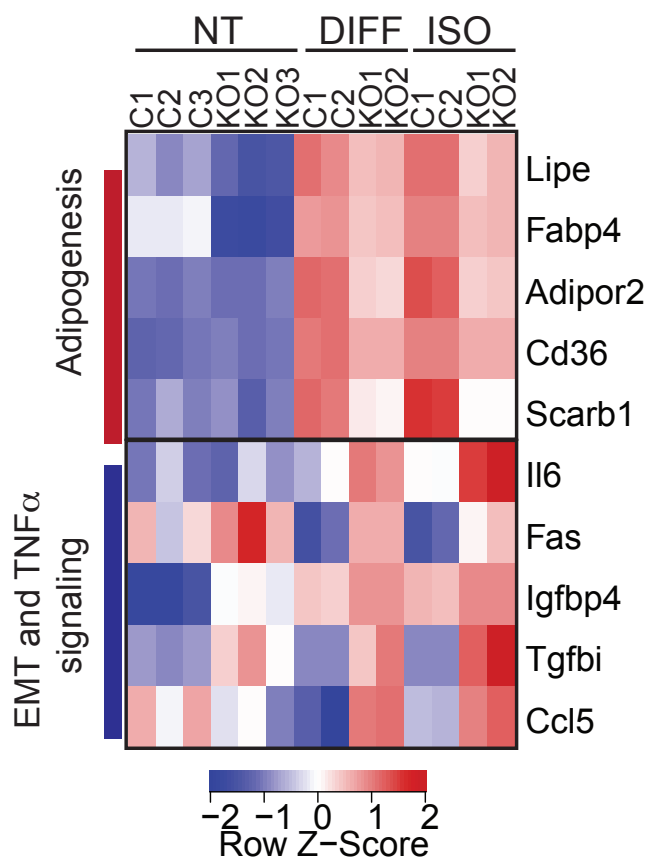

Myc targets
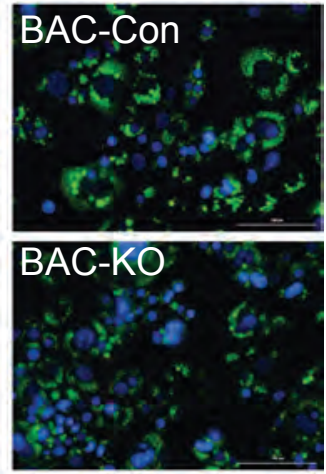
bioRxiv preprint doi: https://doi.org/10.1101/2021.09.13.460063; this version posted September 15,2021 . The copyright holder for this preprint (which was not certified by peer review) is the author/funder, who has granted bioRxiv a license to display the preprint in perpetuity. It is made available under aCC-BY-NC-ND 4.0 International license.

Figure 6

A

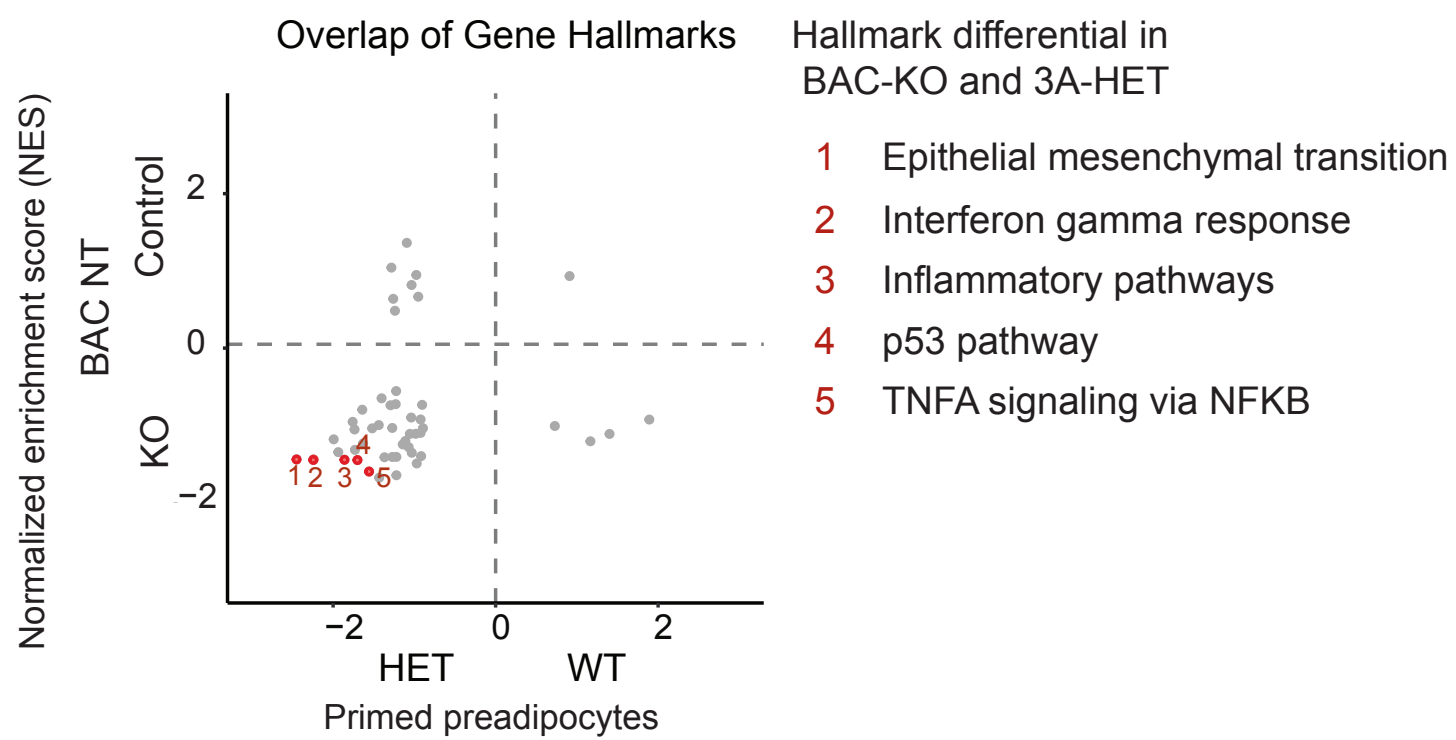

Normalized enrichment score (NES)

B Overlap of Gene Hallmarks

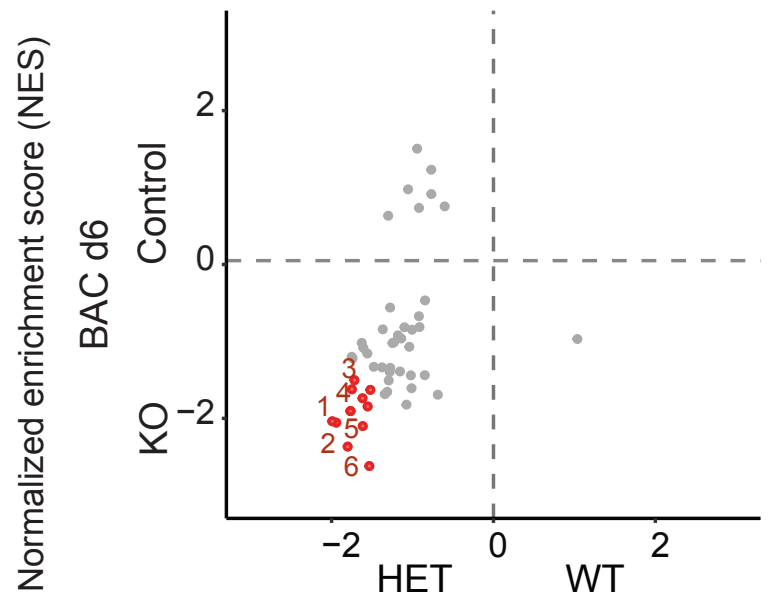

Committed preadipocytes

Normalized enrichment score (NES)

C

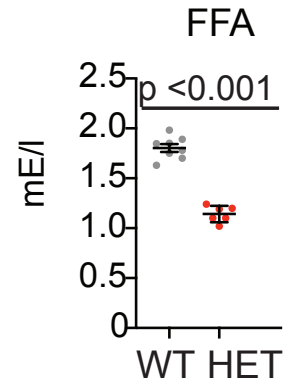

D

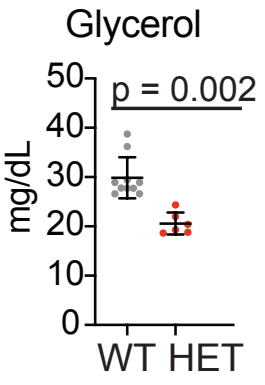

Hallmark differential in BAC-KO and 3A-HET

1 Epithelial mesenchymal transition

2 Interferon gammna response

3 Interferon alpha response

4 Inflammatory pathways

5 TNFA signaling via NFKB

6 IL6 JAK STAT3 signaling
E

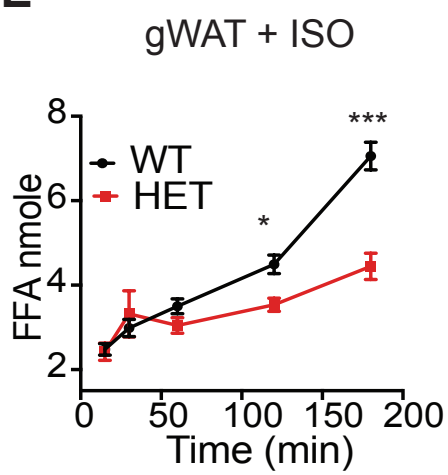

$\mathbf{F}$

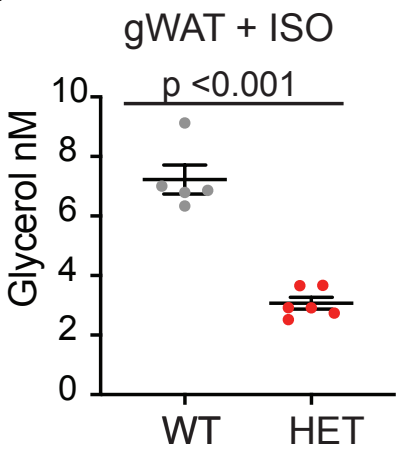


A bioRxiv preprint doi: https://doi.org/10.1101/20 (which was noh68/tified bygpieforeview) is the author/funder, who has granted bioRxiv a license to display the preprin in perpe uity. It is made

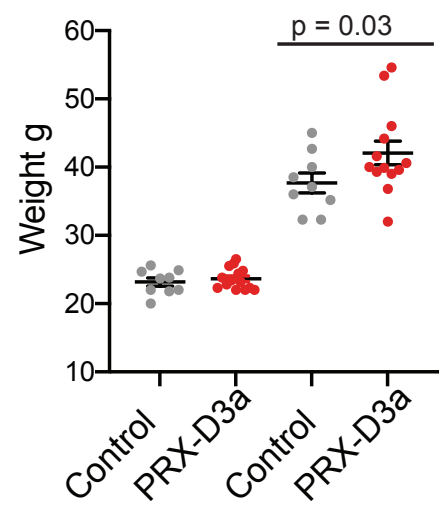
available under -aCC-BY-NC-ND 4.0 International license

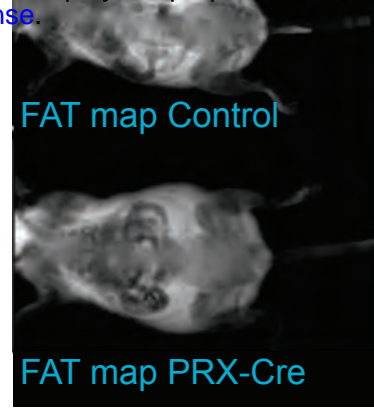

C

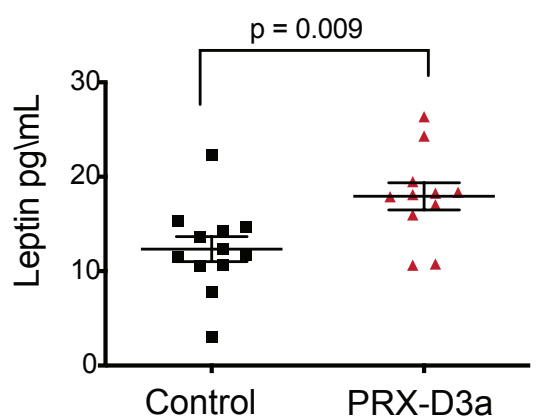

D

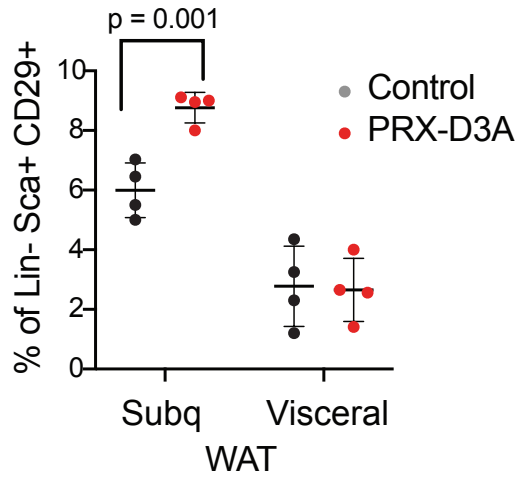

$\mathbf{E}$

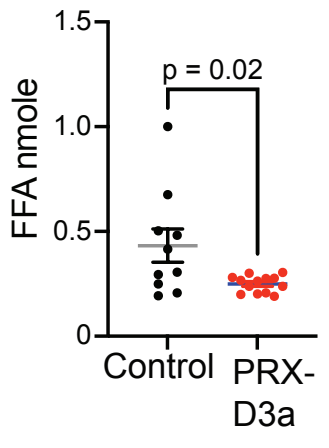


bioRxiv preprint doi: https://doi.org/10.1101/2021.09.13.460063; this version posted September 15, 2021. The copyright holder for this preprint (which was not certified by peer review) is the author/funder, who has granted bioRxiv a license to display the preprint in perpetuity. It is made

a

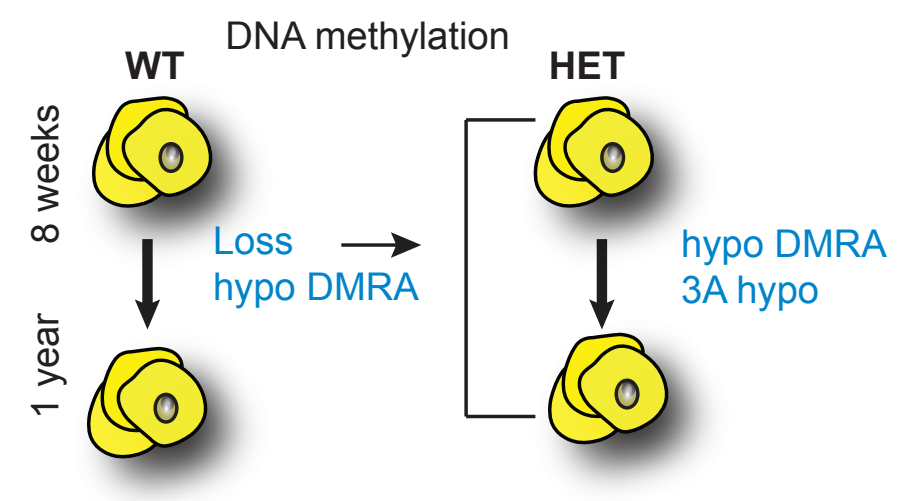

C

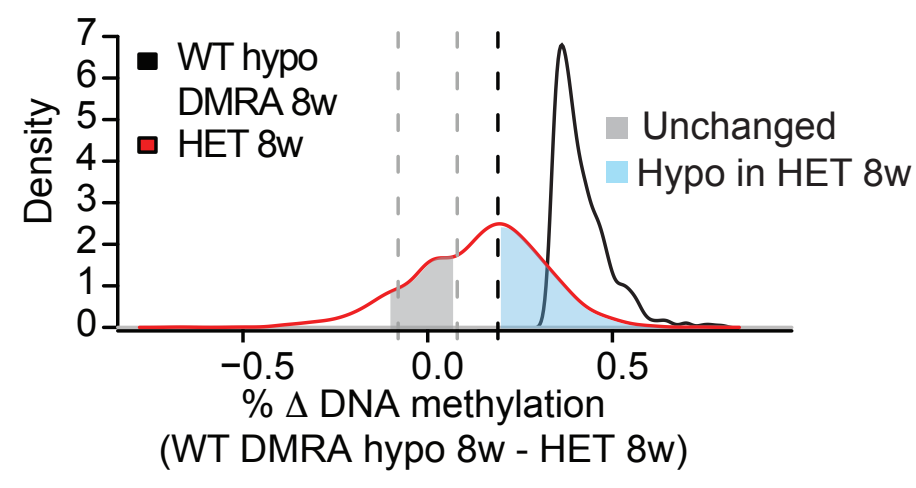

e

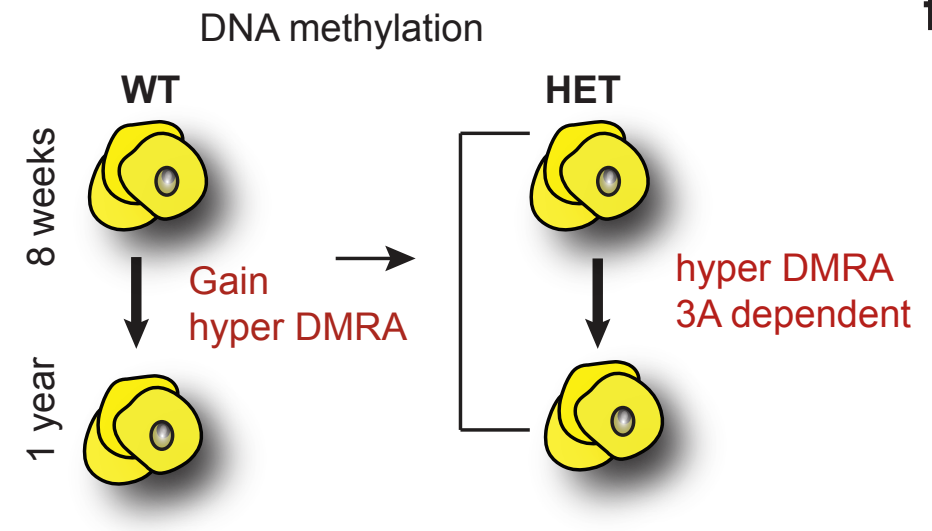

g

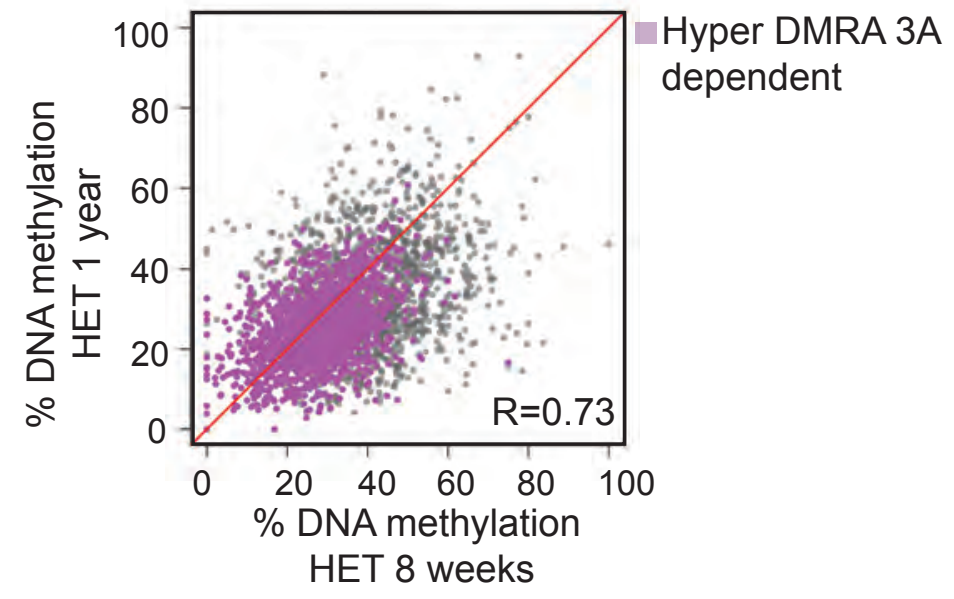

Hypo DMRA

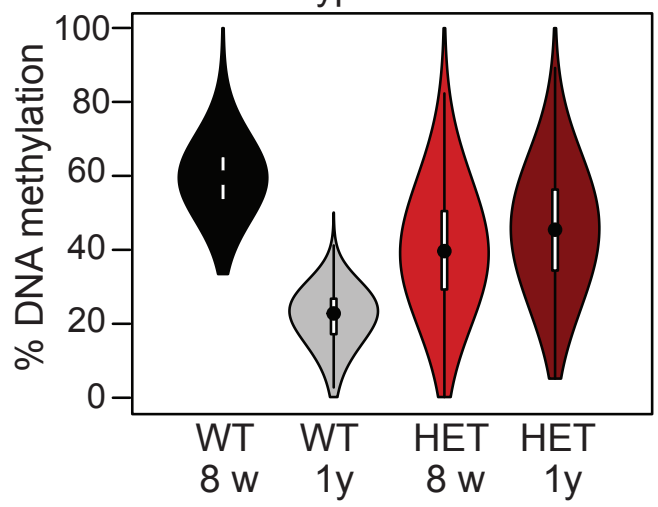

d

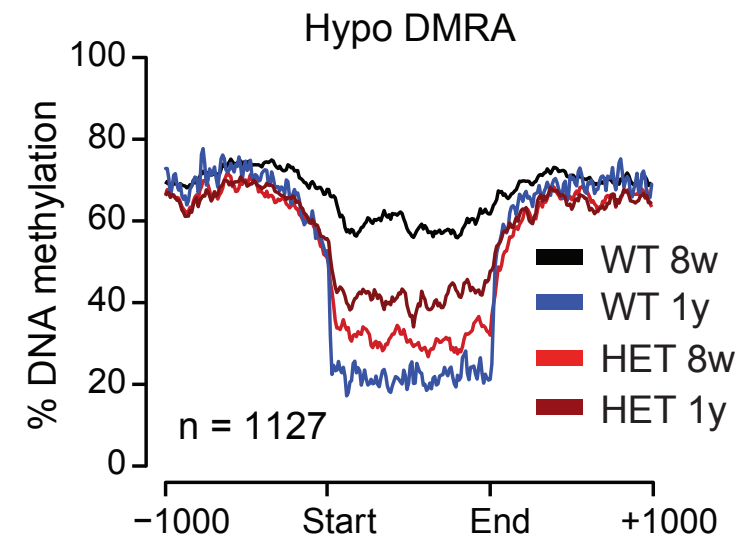

f

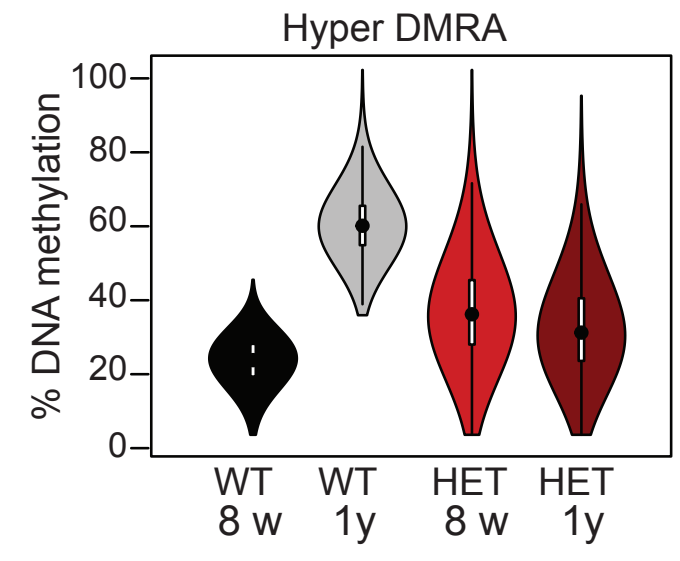


bioRxiv preprint doi: $\mathrm{https}$ //doi.org/10.1101/2021.09.13.460063; this version posted September 15,2021 . The copyright holder for this preprint (which was not certified by peer review) is the author/funder, who has granted bioRxiv a license to display the preprint in perpetuity. It is made

a

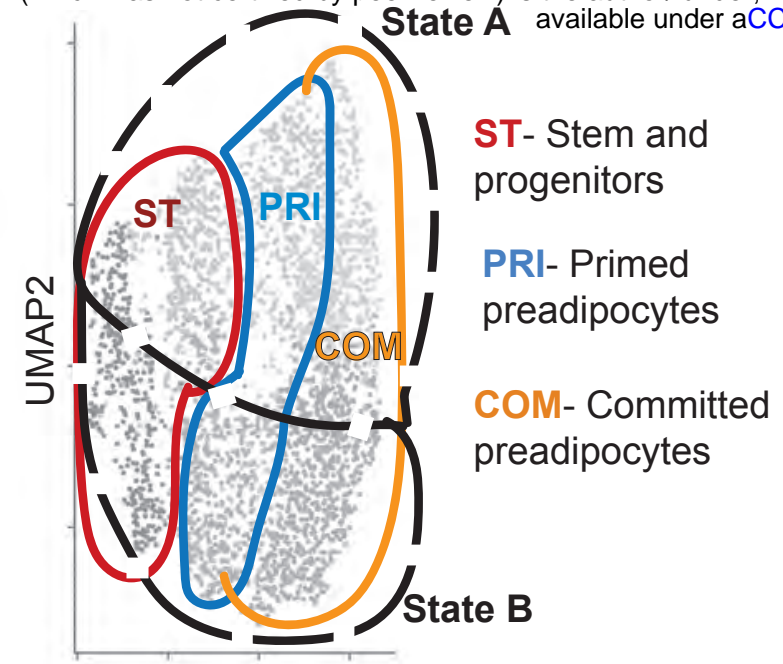

UMAP1

C

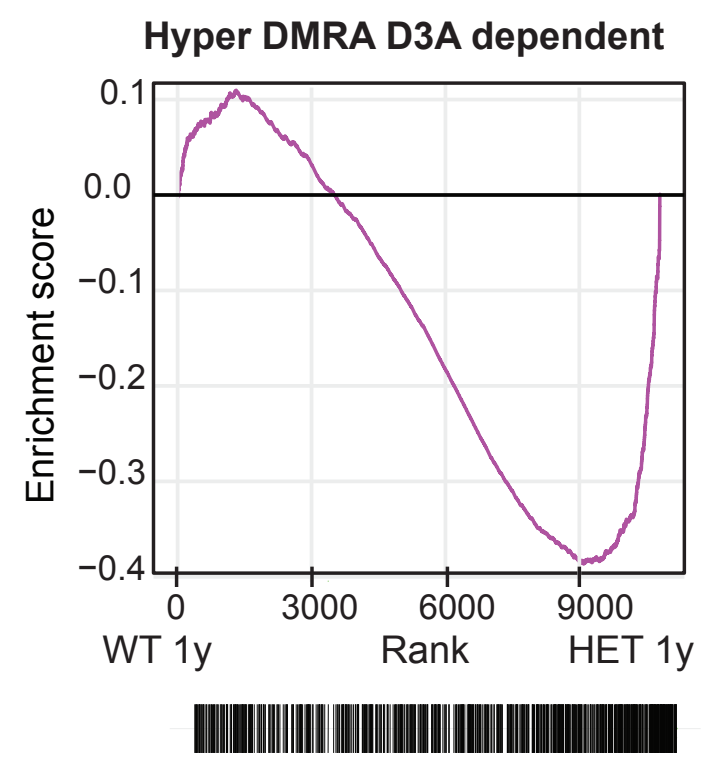

WT vs HET adj. pval $=0.0001$ NES $=-1.83$
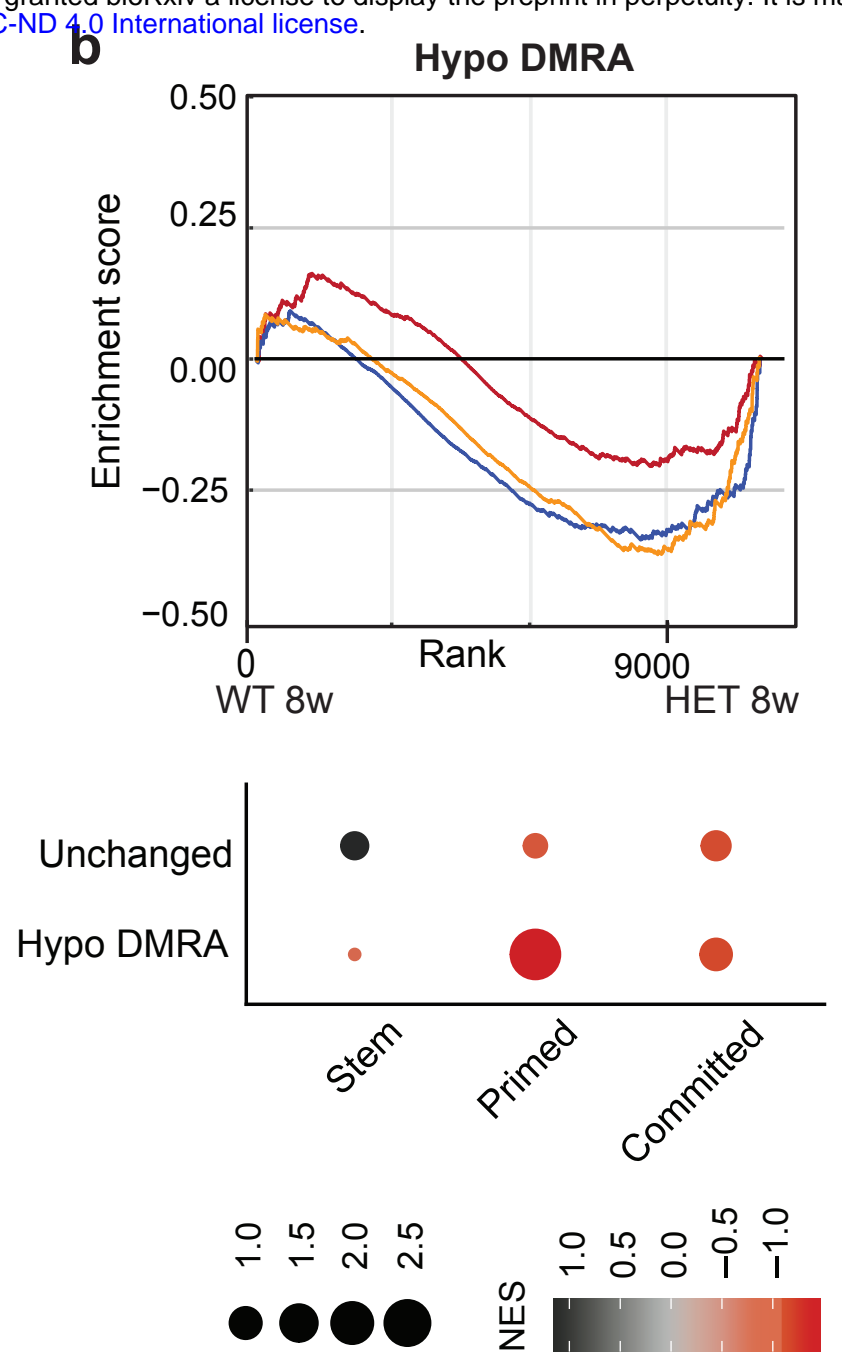

d

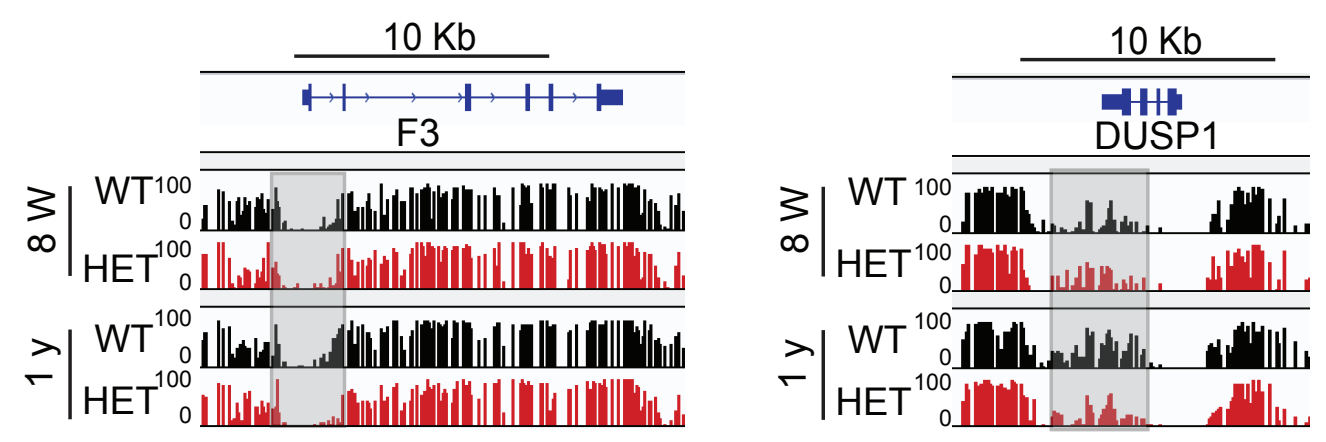

$10 \mathrm{~Kb}$

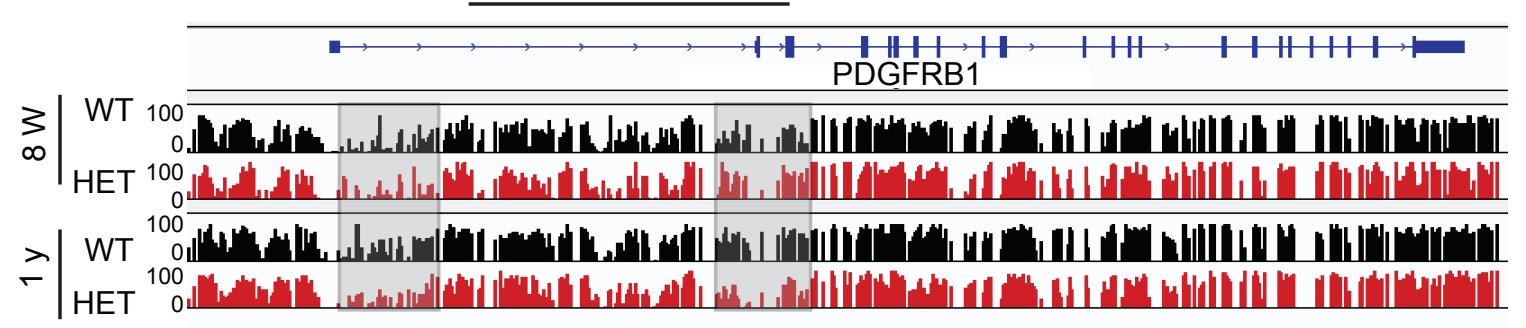

